\title{
Galaxy clustering in 3D and modified gravity theories
}

\author{
D. Munshi, ${ }^{1 \star}$ G. Pratten, ${ }^{1}$ P. Valageas, ${ }^{2,3}$ P. Coles $^{1}$ and P. Brax ${ }^{2,3}$ \\ ${ }^{1}$ Astronomy Centre, School of Mathematical and Physical Sciences, University of Sussex, Brighton BN1 9QH, UK \\ ${ }^{2}$ CEA, IPhT, F-91191 Gif-sur-Yvette, Cedex, France \\ ${ }^{3}$ CNRS, URA, 2306, F-91191 Gif-sur-Yvette, Cedex, France
}

Accepted 2015 November 18. Received 2015 November 16; in original form 2015 August 3

\begin{abstract}
We study Modified Gravity (MG) theories by modelling the redshifted matter power spectrum in a spherical Fourier-Bessel basis. We use a fully non-linear description of the real-space matter power spectrum and include the lowest order redshift-space correction (Kaiser effect), taking into account some additional non-linear contributions. Ignoring relativistic corrections, which are not expected to play an important role for a shallow survey, we analyse two different MG scenarios, namely the generalized Dilaton scalar-tensor theories and the $f(R)$ models in the large curvature regime. We compute the $3 \mathrm{D}$ power spectrum $\mathcal{C}_{\ell}^{\mathrm{s}}\left(k_{1}, k_{2}\right)$ for various such MG theories with and without redshift-space distortions, assuming precise knowledge of background cosmological parameters. Using an all-sky spectroscopic survey with Gaussian selection function $\varphi(r) \propto \exp \left(-r^{2} / r_{0}^{2}\right), r_{0}=150 h^{-1} \mathrm{Mpc}$, and number density of galaxies $\bar{N}=10^{-4} \mathrm{Mpc}^{-3}$, we use a $\chi^{2}$ analysis, and find that the lower order $(\ell \leq 25)$ multipoles of $\mathcal{C}_{\ell}^{\mathrm{s}}\left(k, k^{\prime}\right)$ (with radial modes restricted to $k<0.2 h \mathrm{Mpc}^{-1}$ ) can constraint the parameter $f_{R_{0}}$ at a level of $2 \times 10^{-5}\left(3 \times 10^{-5}\right)$ with $3 \sigma$ confidence for $n=1(2)$. Combining constraints from higher $\ell>25$ modes can further reduce the error bars and thus in principle make cosmological gravity constraints competitive with Solar system tests. However this will require an accurate modelling of non-linear redshift-space distortions. Using a tomographic $\beta(a)$ $m(a)$ parametrization we also derive constraints on specific parameters describing the Dilaton models of MG.
\end{abstract}

Key words: cosmological parameters - cosmology: theory - large-scale structure of Universe.

\section{INTRODUCTION}

The apparent accelerated expansion of the Universe (Ries et al. 1998; Perlmutter et al. 1999) can be explained within General Relativity (GR) by introducing a finely tuned cosmological constant. However, there are alternative explanations for this phenomenon, including Modified Gravity (MG) theories. While the laws of gravity are not well constrained on cosmological scales (Clifton et al. 2013; Joyce et al. 2015), modification of GR are tightly constrained in the Solar system (Ostriker \& Steinhardt 2003) or at astrophysical scales (Jain, Vikram \& Sakestein 2013; Vikram et al. 2013).

As shown by various authors (Bertschinger 2006; Song, Hu \& Sawicki 2007a; Brax et al. 2008) the background dynamics in various dark energy and MG models are nearly indistinguishable. Thus it is important to investigate the evolution of perturbations in these models. The studies of perturbation theory in MG models, in principle, can be classified into two different frameworks: the parametric approach and the non-parametric method, e.g. the principal component analysis (PCA; Zhao et al. 2008, 2009, 2010; Hojjati et al. 2012; Hall, Bonvin \& Challinor 2013). Several parametrizations of MG have been proposed for the evolution of linear perturbations. Such parametrizations typically involve two functions $v(k, a)$ and $\gamma(k, a)$ that both depend on the scalefactor $a$ and wavenumber $k$, when they describe the modification to the Poisson equation for the metric potentials. We will use instead the recently proposed parametrization of the coupling to matter $\beta(a)$ and the mass $m(a)$ of the scalar field, that only depend on the scalefactor (but give rise to both time and scale dependences in the resulting modification to the Poisson equation). The $\beta(a)-m(a)$ offers a unified approach to study various MG models including $f(R)$ gravity and the Symmetron and Dilaton models (Brax, Davis \& Li 2012c). Parametrization based on effective field theory has also been considered (Cheung et al. 2008). Besides the recent progress on the construction of parametrizations, many observational windows have recently been proposed, such as 
the galaxy clustering (Oyazu, Lima \& Hu 2008; Pogosian \& Silvestri 2008), Integrated Sachs-Wolfe (ISW) effect in Cosmic Microwave Background (CMB) anisotropies (Zhang 2006), the galaxy-ISW cross-correlation (Song, Peiris \& Hu 2007b), cluster abundance (Jain \& Zhang 2008; Lombriser et al. 2012), peculiar velocity (Li et al. 2013), redshift-space distortions (RSD; Guzzo et al. 2008 ; Jennings et al. 2012), weak lensing (Heavens, Kitching \& Verde 2007; Schimdt 2008; Tsujikawa \& Tatekawa 2008), 21 cm observations (Hall et al. 2013), matter bispectrum (Gil-Marin et al. 2011), etc. In addition, recently some $N$-body simulation algorithms in MG models have been developed (Li, Mota \& Barrow 2011; Zhao, Li \& Koyama 2011). As shown by various authors (Song et al. 2007b; Lombriser et al. 2012), at the WMAP resolution the modification effects on the CMB mainly come from the ISW effect, which becomes prominent on the superhorizon scales. However, due to the unavoidable cosmic variance on large scales, the constraints from these effects are not significant. On the other hand, since the typical modification scales are in the sub-horizon range, several studies show that the most stringent cosmological constraints come from the large-scale structure data sets. For instance, in the case of $f(R)$ gravity, one obtains $\left|f_{R_{0}}\right| \leq 6.5 \times 10^{-5}$, 95 per cent confidence limit (CL; Dosset, Hu \& Parinson 2014) from the combined analysis of the CMB temperature power spectrum, the galaxy power spectrum and the baryon acoustic oscillation (BAO) measurements, or $\left|f_{R_{0}}\right| \leq 4.6 \times 10^{-5}, 95$ per cent CL (Bel et al. 2015) from the analysis of the galaxy power spectrum through the clustering ratio. This is competitive with Solar system constraints but astrophysical constraints in dwarf galaxies provide tighter bounds $\left|f_{R_{0}}\right| \leq 5 \times 10^{-7}$ (Jain et al. 2013; Vikram et al. 2013).

The past few decades have seen a rapid progress in large-scale galaxy surveys. SDSS $^{1}$ and $2 \mathrm{dFGSS}^{2}$ opened a new horizon in modern cosmology by mapping three-dimensional (3D) positions of millions of galaxies. The Baryon Oscillation Spectroscopic Survey (BOSS) and Dark Energy Survey (DES) represent current state-of-the art galaxy surveys, together with the recently completed WiggleZ. ${ }^{3}$ Future surveys such as the Euclid ${ }^{4}$ (Laureijs et al. 2011), Large Synoptic Survey Telescope (LSST) ${ }^{5}$ and Wide-Field InfraRed Survey Telescope ${ }^{6}$ will measure galaxy clustering with greatly increased statistical power. They will test the theory of GR on cosmological scales. One way to do so is to examine the growth of structure using the $\gamma$-parameter (to be introduced later). Previous calculations suggest that Euclid can constrain $\gamma$ to 0.01 (for $\Lambda$ cold dark matter $-\Lambda \mathrm{CDM}-\gamma$ corresponds to $\gamma \simeq 0.55$; Linder 2005; Heavens et al. 2007). Generic modification of gravity may require more than just one parameter (Amendola, Kunz \& Sapone 2008; Ferreira \& Skordis 2010) but previous studies have shown that a Euclid-type survey could measure these parameters to high precision (Daniel et al. 2010; Amendola et al. 2011).

The power spectrum of density inhomogeneities in the nearby Universe and the temperature fluctuations in the CMB sky carry the bulk of the cosmological information and are routinely employed in analysing data from CMB experiments as well as large-scale structure (LSS) surveys. They carry complementary cosmological information; all-sky CMB observations such as NASA's WMAP ${ }^{7}$ and ESA's Planck ${ }^{8}$ experiments primarily probe the distribution of matter and radiation at redshift $z=1300$. Such surveys are projected or $2 \mathrm{D}$ surveys. The large-scale surveys such as those obtained with ESA's Euclid mission will give us a window at a lower redshift range $z \approx 0-2$ and are designed to provide a 3D map of the Universe using more than 50 million galaxies with spectroscopic redshifts. There are other LSS surveys that are either being planned or in various stages of developments e.g. Rassat et al. (2005) for DES, ${ }^{9}$ Tyson \& LSST (2004) for LSST and Schlegel et al. (2007) for BOSS. ${ }^{10}$ These surveys are designed to map the local dark Universe using weak lensing of galaxies as well as studying baryonic oscillation features in the matter power spectrum. This has motivated in recent years a flurry of activity in developing 3D power-spectrum analysis for cosmological data using spherical Fourier-Bessel (sFB) decomposition (see e.g. Heavens \& Taylor 1995; Castro, Heavens \& Kitching 2005; Erdogue et al. 2006; Abramo, Reimberg \& Xavier 2010; Asrorey et al. 2012; Lanuss, Rassat \& Strack 2012; Rassat \& Refregier 2012; Shapiro, Crittenden \& Percival 2012; Pratten \& Munshi 2013).The sFB power spectrum recovers the 3D power spectrum at each wavenumber $k$ and its angular dependence is encoded in the angular momentum $\ell$.

In this paper we use the results derived in Pratten \& Munshi (2013), to derive the 3D density power spectrum for models of MG theories. The primary goal is to check to what extent such power-spectrum analysis can be used to constrain departure from GR using future redshift surveys.

This paper is organized as follows: in Section 2 we review different types of MG theories with special attention to $f(R)$ gravity theories as well as the models known as the generalized Dilaton models. We introduce the 3D power spectrum in Section 3 using an sFB transform, which provides a natural framework for treating RSD. For the specific baseline $\Lambda$ CDM cosmology we take the following parameter values: $h=0.73, \Omega_{\mathrm{M}}=0.24, \Omega_{\mathrm{DE}}=0.76, \Omega_{\mathrm{K}}=0, w_{\mathrm{DE}}=-1, \sigma_{8}=0.76, n_{\mathrm{s}}=0.958$.

\section{MG THEORIES}

Henceforth, we consider MG theories that differ from the standard $\Lambda$ CDM cosmology by adding a new degree of freedom, which can be associated to a scalar field $\varphi$. This new component can drive the accelerated expansion of the Universe at late times, typically through the

\footnotetext{
${ }^{1}$ http://www.sdss.org/

${ }^{2}$ http://www.roe.ac.uk/ jap/2df/

${ }^{3} \mathrm{http} / / /$ wigglez.swin.edu.au/site/

${ }^{4} \mathrm{http}: / /$ sci.esa.int/euclid

${ }^{5}$ http://www.lsst.org/lsst/

${ }^{6} \mathrm{http} / / /$ wirst.gsfc.nasa.gov/

${ }^{7}$ http://map.gsfc.nasa.gov/

${ }^{8}$ http://www.rssd.esa.int/planck

${ }^{9} \mathrm{http}: / /$ www.darkenergysurvey.org/

${ }^{10} \mathrm{http} / / / \cos m o l o g y . l b l . g o v / B O S S /$
} 
non-zero value of the minimum of its potential, although this can be seen as introducing a simple cosmological constant. The main difference from the $\Lambda \mathrm{CDM}$ cosmology and quintessence scenarios, which only modify the background expansion, is that the fifth force induced by the scalar field also modifies the growth of structures, typically giving a new scale dependence to the linear growing modes of density perturbations and accelerating the collapse of overdense regions.

These MG theories can be classified in three broad categories: Chameleon, K-mouflage and Vainshtein scenarios, according to their non-linear screening mechanism that ensures convergence to GR in small-scale and high-density environments such as the Solar system (as the high-accuracy Solar-system measurements provide tight constraints on local deviations from GR). The K-mouflage and Vainshtein mechanisms (Vainshtein 1972) rely on non-standard kinetic terms that drive spatial gradients of the scalar field to zero in high-density regions and suppress the fifth force. Chameleon scenarios typically contain additional couplings between the scalar field and the metric, or new geometric terms beyond the Einstein-Hilbert gravitational action. These two equivalent descriptions can be captured by the $\{\beta(a), m(a)\}$ parametrization which will be used throughout this work (Brax et al. 2012b,c).

In this paper, we focus on two different models of the Chameleon type. In the Dilaton models, the coupling between the scalar field and the metric depends on the scalar field value, so that in dense environments where the scalar field is driven to zero, the coupling also vanishes, which suppresses the fifth force as in the Damour-Polyakov mechanism (Damour \& Polyakov 1994). In the $f(R)$ models, the coupling of the scalar field to matter is constant but its effective potential depends on the environment and its mass becomes large in high-density regions. This suppresses the magnitude of the fifth force through a Yukawa screening. In all chameleon cases, these modifications of gravity induce a global enhancement of the effective force of gravity, due to the fifth force, which directly translates into an increase of structure formation. In this section, we review the Dilaton and the $f(R)$ models and we describe parametrization in the context of large-scale structure formation.

\subsection{Gravity in Dilaton models}

The Dilaton theories of MG are chameleon models with the Damour-Polyakov property (Damour \& Polyakov 1994), according to which the coupling between the scalar field $\varphi$ and the rest of the matter components approaches zero in dense environments (Pietroni 2005; Olive \& Pospelov 2008; Hinterbichler \& Khoury 2010). In contrast with the $f(R)$ theories described in Section 2.2 below, the scalar field here takes on a small mass everywhere and thus mediates a long-range (screened) force. These Dilaton models are scalar-tensor theories, where the action defining the system takes the general form

$S=\int \mathrm{d}^{4} x \sqrt{-g}\left[\frac{M_{\mathrm{Pl}}^{2}}{2} R-\frac{1}{2}(\nabla \varphi)^{2}-V(\varphi)-\Lambda_{0}^{4}\right]+\int \mathrm{d}^{4} x \sqrt{-\tilde{g}} \tilde{\mathcal{L}}_{\mathrm{m}}\left(\psi_{\mathrm{m}}^{(i)}, \tilde{g}_{\mu \nu}\right)$,

where $M_{\mathrm{Pl}}=\left(8 \pi \mathcal{G}_{\mathrm{N}}\right)^{-1 / 2}$ is the reduced Planck mass (in natural units), $g$ is the determinant of the Einstein-frame metric tensor $g_{\mu \nu}$ and $\tilde{g}$ the determinant of the Jordan-frame metric tensor $\tilde{g}_{\mu \nu}$, which is given by the conformal rescaling

$\tilde{g}_{\mu \nu}=A^{2}(\varphi) g_{\mu \nu}$.

The various matter fields $\psi_{\mathrm{m}}^{(i)}$ are governed by the Jordan-frame Lagrangian density $\tilde{\mathcal{L}}_{\mathrm{m}}$ and the scalar field $\varphi$ by the Einstein-frame Lagrangian density $\mathcal{L}_{\varphi}=-1 / 2(\nabla \varphi)^{2}-V(\varphi)$, with the scalar-field potential $V(\varphi)$. There is no explicit coupling between matter and the scalar field and the fifth force on matter particles due to the scalar field arises from the conformal transformation (2) (more precisely, through gradients of $A$ ). In the Lagrangian presented in equation (1) we explicitly wrote the cosmological constant term $\Lambda_{0}^{4}$, so that the minimum of $V(\varphi)$ is zero, which is reached for $\varphi \rightarrow \infty$, but this could also be interpreted as the non-zero minimum of the scalar field potential.

In the original Dilaton model, the potential of the scalar field $V(\varphi)$ and its coupling $A(\varphi)$ with the metric have the following functional forms:

$V(\varphi)=V_{0} \exp \left(-\frac{\varphi}{M_{\mathrm{Pl}}}\right)$

$A(\varphi)=1+\frac{A_{2}}{2} \frac{\varphi^{2}}{M_{\mathrm{Pl}}^{2}}$,

where $\left\{V_{0}, A_{2}\right\}$ are the two free parameters. In dense regions where $\varphi \approx 0$, the coupling to matter is negligible, and gravity converges to GR. However, the field nevertheless mediates a long range gravitational force that has an effect elsewhere, in less dense environments. This model can be generalized to a greater class of Dilaton models, by keeping the coupling function as in equation (4) but considering more general potentials. Then, instead of specifying the model by its potential $V(\varphi)$ it is convenient to define the model by the tomographic parametrization $\{\beta(a), m(a)\}$ (Brax et al. 2012c; Brax \& Valageas 2013), in terms of the scalefactor $a(t)$, where the coupling $\beta(a)$ and the scalar field mass $m(a)$ are defined as

$\beta(a) \equiv \beta[\bar{\varphi}(a)]=M_{\mathrm{Pl}} \frac{\mathrm{d} \ln A}{\mathrm{~d} \varphi}(\bar{\varphi})$,

$m^{2}(a) \equiv m^{2}[\bar{\varphi}(a), \bar{\rho}(a)]=\frac{1}{c^{2}}\left[\frac{\mathrm{d}^{2} V}{\mathrm{~d} \varphi^{2}}(\bar{\varphi})+\bar{\rho} \frac{\mathrm{d}^{2} A}{\mathrm{~d} \varphi^{2}}(\bar{\varphi})\right]$. 
Table 1. Parameters describing the Dilaton models considered in our study. The parameters are used to define the scalar potential $V(\varphi)$ and the coupling function $A(\varphi)$ through the $\{\beta(a), m(a)\}$ parametrization defined in equations (5)-(8).

\begin{tabular}{lcccc}
\hline Model & $m_{0}\left(h \mathrm{Mpc}^{-1}\right)$ & $r$ & $\beta_{0}$ & $s$ \\
\hline (A1,A2,A3) & $(0.334,0.334,0.334)$ & $(1.0,1.0,1.0)$ & $(0.5,0.5,0.5)$ & $(0.6,0.24,0.12)$ \\
(B1,B3,B4) & $(0.334,0.334,0.334)$ & $(1.0,1.0,1.0)$ & $(0.25,0.75,1.0)$ & $(0.24,0.24,0.24)$ \\
(C1,C3,C4) & $(0.334,0.334,0.334)$ & $(1.33,0.67,0.4)$ & $(0.5,0.5,0.5)$ & $(0.24,0.24,0.24)$ \\
(E1,E3,E4) & $(0.667,0.167,0.111)$ & $(1.0,1.0,1.0)$ & $(0.5,0.5,0.5)$ & $(0.24,0.24,0.24)$ \\
\hline
\end{tabular}

In this paper we consider the simple forms

$m(a)=m_{0} a^{-r}, \quad \beta(a)=\beta_{0} \exp \left[-s \frac{a^{2 r-3}-1}{3-2 r}\right]$,

with

$s=\frac{9 A_{2} \Omega_{\mathrm{m} 0} H_{0}^{2}}{c^{2} m_{0}^{2}}$.

(The exponential potential equation 3 corresponds to $r=3 / 2$.) The values of the free parameters $\left\{m_{0}, r, \beta_{0}, s\right\}$ that enter equation (7) are displayed in Table 1. The models $\{\mathrm{A}, \mathrm{B}, \mathrm{C}\}$ were chosen such as to correspond to those studied in Brax \& Valageas (2013) and Brax et al. (2012a), where detailed comparisons between numerical and analytical calculations are presented. The models $\{$ A,B,C,E $\}$ probe the dependence on $\left\{s, \beta_{0}, r, m_{0}\right\}$, respectively, other parameters being fixed (instead of the models 'D' considered in Brax \& Valageas 2013 , which probe the dependence on $m_{0}$ at fixed $A_{2}$, we introduced the models E that probe the dependence on the parameter $m_{0}$ at fixed $\left\{s, \beta_{0}\right.$, $r\}$ ). These models probe deviations from the $\Lambda \mathrm{CDM}$ cosmology of less than 20 per cent, in terms of the matter power spectrum.

In these Dilaton models, the coupling function $A$ is always very close to unity, so that most Einstein-frame and Jordan-frame quantities (e.g. Hubble expansion rates or densities) are almost identical. Indeed, from equations (4), (5) and (8) we obtain $\bar{A} \simeq 1+\beta^{2} /\left(2 A_{2}\right)$ and $A_{2} \sim\left(\mathrm{cm}_{0} / H_{0}\right)^{2}$. Solar system tests of gravity imply that $m_{0} \gtrsim 10^{3} H_{0} / c$, whence $A_{2} \gtrsim 10^{6}, H / c m \lesssim 10^{-3}$ and $|\bar{A}-1| \lesssim 10^{-6}$. Therefore, the Jordan-frame and Einstein-frame scalefactors and background matter densities, related by $\tilde{a}=\bar{A} a$ and $\tilde{\bar{\rho}}=\bar{A}^{-4} \bar{\rho}$, can be considered equal, as well as the cosmic times and Hubble expansion rates. (However, in this section we work in the Einstein frame, where the analysis of the gravitational dynamics is simpler.)

In the Einstein frame, the Friedmann equation takes the usual form,

$3 M_{\mathrm{Pl}}^{2} H^{2}=\bar{\rho}+\bar{\rho}_{\varphi}+\bar{\rho}_{\Lambda}$,

where we consider the matter and scalar field components and the cosmological constant contribution $\bar{\rho}_{\Lambda}$. One can check that the scalar field energy density is negligible as compared with the matter density, $\bar{\rho}_{\varphi} / \bar{\rho} \sim 10^{-6}$, so that the Friedmann equation (9) is governed by the matter density and the cosmological constant and we recover the $\Lambda$ CDM cosmological expansion, $3 M_{\mathrm{Pl}}^{2} H^{2}=\bar{\rho}+\bar{\rho}_{\Lambda}$, up to an accuracy of $10^{-6}$. In the Newtonian gauge, the perturbed metric can be written as

$\mathrm{d} s^{2}=-(1+2 \Phi) \mathrm{d} t^{2}+a^{2}(t)(1-2 \Psi) \delta_{i j} \mathrm{~d} x^{i} \mathrm{~d} x^{j}$,

where $\Phi$ and $\Psi$ are the Einstein-frame metric gravitational potentials. One can check that the impact of the scalar field fluctuations on the metric potentials are again negligible, as $\left|\delta \rho_{\varphi}\right| /|\delta \rho| \lesssim 10^{-6}$, and we have within a $10^{-6}$ accuracy $\Phi=\Psi=\Psi_{\mathrm{N}}$, where $\Psi_{\mathrm{N}}$ is the Newtonian potential given by the Poisson equation,

$\frac{\nabla^{2}}{a^{2}} \Psi_{\mathrm{N}}=4 \pi \mathcal{G}_{\mathrm{N}} \delta \rho=\frac{3 \Omega_{\mathrm{m} 0} H_{0}^{2}}{2 a^{3}} \delta$,

where $\delta=\delta \rho / \bar{\rho}$ is the matter density contrast. However, the dynamics of matter particles are modified by the scalar field, which gives rise to a fifth force given by $\boldsymbol{F}_{A}=-c^{2} \nabla \ln A$, that is, in the Euler equation we must add to the Newtonian potential $\Psi_{\mathrm{N}}$ a fifth-force potential $\Psi_{A}=c^{2} \ln A$ that is not negligible and can lead to 10 per cent deviations to the matter density power spectrum for the parameters given in Table 1 (indeed, whereas $|A-1| \lesssim 10^{-6}$ is negligible as compared with unity, it is not negligible as compared with $\left|\Psi_{\mathrm{N}}\right| / c^{2} \lesssim 10^{-5}$ ).

\subsection{Gravity in $f(R)$ theories}

In models known as the $f(R)$ gravity, the Einstein-Hilbert action $S_{\mathrm{GR}}$ is modified by promoting the Ricci scalar $R$ to a function of $R$ (Buchdahl 1970; Starobinsky 1980, 2007; Hu \& Sawicki 2007a). The new action $S$ for the $f(R)$ gravity theories can be written as

$S=\int \mathrm{d}^{4} x \sqrt{-g}\left[\frac{M_{\mathrm{Pl}}^{2}}{2}[R+f(R)]-\Lambda_{0}^{4}+\mathcal{L}_{\mathrm{m}}\left(\psi_{\mathrm{m}}^{(i)}\right)\right]$,

where we explicitly wrote the cosmological constant contribution $\Lambda_{0}^{4}$, although it is often included within the function $f(R)$ [with our choice $f(R)$ describes the deviations from GR and from the $\Lambda$ CDM cosmology]. In this section, contrary to the previous Section 2.1 where we studied 
Dilaton models, we denote with a tilde Einstein-frame quantities instead of Jordan-frame ones, because we now work in the Jordan frame. In the parametrization of $\mathrm{Hu} \&$ Sawicki (2007a), the functional form $f(R)$ can be expressed in the high curvature limit as

$f(R)=-\frac{f_{R_{0}}}{n} \frac{R_{0}^{n+1}}{R^{n}}, \quad f_{R} \equiv \frac{\mathrm{d} f(R)}{\mathrm{d} R}=f_{R_{0}} \frac{R_{0}^{n+1}}{R^{n+1}}$.

The two independent parameters, $f_{R_{0}}<0$ and $n>0$, can be constrained by observations. In the above expression, $R_{0}$ is the present value of the Ricci scalar for the cosmological background. Note that this parametrization and that of Starobinsky (2007) both reproduce the same results in the large curvature regime.

The $f(R)$ theories of gravity invoke the Chameleon mechanism to screen modifications of GR in dense environments such as in our Solar system. In this model, this occurs by requiring that all extra terms vanish in high curvature environment, i.e. by requiring $f\left(|R| \gg\left|R_{0}\right|\right) \rightarrow 0$. In such a theory, the background expansion follows the $\Lambda$ CDM dynamics (as observational constraints imply $\left|f_{R_{0}}\right| \ll 1$ ), and the growth of structure is only affected on intermediate and quasi-linear scales.

There is an essential connection between the formulation of the $f(R)$ theory presented above, and scalar-tensor theories of MG. Upon the coordinate rescaling $\tilde{g}_{\mu \nu}=A^{-2}(\varphi) g_{\mu \nu}$ (recall that in this section $\tilde{g}_{\mu \nu}$ is the Einstein-frame metric), with $A(\varphi)=\exp \left[\beta \varphi / M_{\mathrm{Pl}}\right]$ and $\beta=1 / \sqrt{6}$, the $f(R)$ modifications to GR are recast as arising from contributions of an extra scalar field $\varphi$, that is subject to a potential $V(\varphi)$ given by

$V(\varphi)=\frac{M_{\mathrm{Pl}}^{2}}{2}\left(\frac{R f_{R}-f(R)}{\left(1+f_{R}\right)^{2}}\right), \quad f_{R}=\exp \left[-\frac{2 \beta \varphi}{M_{\mathrm{Pl}}}\right]-1$.

In that sense, $f(R)$ theories are equivalent to a scalar-tensor theory expressed in the Einstein frame (Chiba 2003; Nunez \& Solgnaik 2004). In this new formulation, the screening mechanism takes another form: the mass of the scalar field grows with matter density, and a Yukawa-like potential suppresses the fifth force in dense environments. This can be conveniently reformulated by saying that screening takes place wherever the scalar field is small compared to the ambient Newtonian potential.

It turns out that all chameleon-like models such as $f(R)$ theories can again be parametrized by the value of the mass $m(a)$ and the coupling $\beta(a)$ of the scalar field, in terms of the scalefactor $a$ and the associated background matter density $\bar{\rho}(a)=3 \Omega_{\mathrm{m} 0} H_{0}^{2} M_{\mathrm{Pl}}^{2} / a^{3}$. With the specific functional form of $f(R)$ given by equation (13), we can directly relate $\left\{n, f_{R_{0}}\right\}$ to $\{\beta(a), m(a)\}$ via

$m(a)=m_{0}\left(\frac{4 \Omega_{\Lambda 0}+\Omega_{\mathrm{m} 0} a^{-3}}{4 \Omega_{\Lambda 0}+\Omega_{\mathrm{m} 0}}\right)^{(n+2) / 2}, \quad m_{0}=\frac{H_{0}}{c} \sqrt{\frac{\Omega_{\mathrm{m} 0}+4 \Omega_{\Lambda 0}}{(n+1)\left|f_{R 0}\right|}}, \quad \beta(a)=\frac{1}{\sqrt{6}}$.

In this paper, we consider values of $n=\{1,2\}$ and $\left|f_{R 0}\right|=\left\{10^{-4}, 10^{-5}, 10^{-6}\right\}$. The larger value of $\left|f_{R 0}\right|$ is currently ruled out by other independent probes, so this serves as a consistency test.

As for the Dilaton models described in Section 2.1, the $f(R)$ models that we consider in this paper follow very closely the $\Lambda$ CDM cosmology at the background level, because $\left|f_{R_{0}}\right| \ll 1$. Indeed, from the action (12) one obtains the Friedmann equation as

$3 M_{\mathrm{Pl}}^{2}\left[H^{2}-\bar{f}_{R}\left(H^{2}+\dot{H}\right)+\bar{f} / 6+\bar{f}_{R R} H \dot{\bar{R}}\right]=\bar{\rho}+\bar{\rho}_{\Lambda}$,

where the dot denotes the derivative with respect to cosmic time $t$ and $f_{R R}=\mathrm{d}^{2} f / \mathrm{d} R^{2}$. In the background we have $\bar{R}=12 H^{2}+6 \dot{H}$ and we can check that all extra terms in the brackets in equation (16) are of order $\left|f_{R_{0}}\right| H^{2}$, so that we recover the $\Lambda \mathrm{CDM}$ expansion, $3 M_{\mathrm{Pl}}^{2} H^{2}=\bar{\rho}+\bar{\rho}_{\Lambda}$, up to an accuracy of $10^{-4}$ for $\left|f_{R_{0}}\right| \lesssim 10^{-4}$. Moreover, the conformal factor $A(\varphi)$ is given by $A=\left(1+f_{R}\right)^{-1 / 2}$, so that $|\bar{A}-1| \lesssim 10^{-4}$ and the background quantities associated with the Einstein and Jordan frames can be considered equal, and equal to the $\Lambda$ CDM reference, up to an accuracy of $10^{-4}$. Considering the metric and density perturbations, we can again write the Newtonian gauge metric as in equation (10) (but this is now the Jordan-frame metric). Then, in the small-scale sub-horizon limit, the modified Einstein equations lead to

$\Phi=\Psi_{\mathrm{N}}-\frac{c^{2}}{2} \delta f_{R}, \quad \Psi=\Psi_{\mathrm{N}}+\frac{c^{2}}{2} \delta f_{R}$,

where $\delta f_{R}=f_{R}-\bar{f}_{F}$ and $\Psi_{\mathrm{N}}$ is the Newtonian gravitational potential defined as in GR by equation (11). Thus, because we work in the Jordan frame, in contrast with the Dilaton case presented in Section 2.1, the modification of gravity directly appears through the metric potentials equation (17). Finally, the dynamics of the matter particles are given by the geodesic equation, or the Euler equation in the large-scale single-stream limit, where the Newtonian potential that appears in GR is replaced by the potential $\Phi$ given in equation (17).

\subsection{MG and structure formation}

\subsubsection{Impact of MG on 3D matter clustering}

As we have seen in the previous sections, Dilatons and $f(R)$ theories reproduce the smooth background expansion history of the standard $\Lambda \mathrm{CDM}$ cosmology (up to an accuracy of $10^{-4}$ or better that is sufficient for our purposes). To distinguish between, and hopefully test such competing gravitational theories it is thus necessary to analyse the evolution of matter or metric perturbations. To lowest order in cosmological perturbations, non-standard gravitational scenarios effectively result in a time- and scale-dependent modification of the Newtonian constant $\mathcal{G}_{\mathrm{N}}$. In the non-linear regime, the modifications become more complex as they become sensitive to the screening mechanism, which depends non-linearly on the environment and modifies the form of the equations of motion (e.g. the effective Poisson equation is no longer linear). 
These modifications induce a distortion of the dynamical as well as statistical properties of the matter clustering statistics. To this end, we focus on the signature of these effects on the matter density power spectrum $P(k, z)$, or more precisely its expansion $\mathcal{C}_{\ell}(k)$ on spherical multipoles, which is well suited to the cosmological analysis of wide galaxy surveys. (We also include RSD through a simple approximation that is exact at linear order and includes some non-linear contributions.)

Thus, we need a way of modelling of the matter power spectrum that applies to the standard $\Lambda$ CDM scenario as well as these MG models. We use the approach developed in Valageas, Nishimichi \& Taruya (2013), for the $\Lambda$ CDM cosmology, and next extended to these MG cosmologies in Brax \& Valageas (2013). This method combines the results from one-loop perturbation theory with that from halo model predictions. By construction, this power spectrum agrees with perturbation theory up to order $P_{\mathrm{L}}^{2}$. In the $\Lambda$ CDM cosmology, this corresponds to the standard one-loop diagrams Bernardeau et al. (2002). In the MG models, the linear propagators and the vertices are modified, with new scale and time dependences. In addition, the effective Poisson equation, obtained after integrating over the scalar field, becomes non-linear. At one-loop order, this gives rise to a new diagram for the power spectrum. Beyond the perturbative regime, predictions from a suitably altered halo model are incorporated in the high- $k$ limit. The impact of the MG in the non-linear dynamics is taken into account through the halo mass function but the impact of the MG on the halo profiles is ignored, i.e. we keep the NFW profile from Navarro, Frenk \& White (1996) and the mass-concentration relation from Valageas et al. (2013). The resulting matter density power spectrum $P_{\delta \delta}(k)$ has been tested against numerical simulations and found to be in agreement for the entire available $k$ range from simulations $k<3 h^{-1}$ Mpc (Brax \& Valageas 2013).

\subsubsection{Numerical results for the linear and non-linear $3 D$ power spectra}

In the MG scenarios, the linear growing mode $D_{+}(k, t)$ and the linear growth rate $f(k, t)=\partial \ln D_{+} / \partial \ln a$ become scale dependent. We show the linear growth rates $f(k, z)$ in Fig. 1 for the $f(R),(n=1)$, models and in Fig. 2 for the Dilaton models. For the Dilaton models, we only show those models that exhibit maximal departure from GR predictions. The MG models that we consider in this paper amplify and accelerate the growth of large-scale structures, so that their linear growth rates are greater than the $\Lambda \mathrm{CDM}$ one. The relative importance of the fifth force also increases with time and is mostly relevant in the dark energy era, at $z \lesssim 2$. In addition, we can see in Figs 1 and 2 the scale dependence generated by these MG models. The deviation from the $\Lambda$ CDM growth rates decreases along with $\left|f_{R_{0}}\right|$. For the dilaton models the deviation decreases on these scales as $\mathrm{B} 4>\mathrm{A} 3>\mathrm{E} 3>\mathrm{C} 1$.

The non-linear matter power spectra for the MG theories are displayed in Fig. 3. The deviations from the $\Lambda$ CDM power spectrum peak at the weakly non-linear scales $k \sim 5 h \mathrm{Mpc}^{-1}$, due to the amplification by the non-linear dynamics. Moreover, at very large scales (much beyond the Compton wavelength) all models converge back to GR and to the $\Lambda$ CDM cosmology. At high $k, k \gtrsim 10 h$ Mpc ${ }^{-1}$, we may underestimate the deviation from the $\Lambda \mathrm{CDM}$ power spectrum because we neglect the impact of the MG on the halo profiles. However, the analysis presented in this paper is restricted to linear or weakly non-linear scales, $k \lesssim 0.2 h \mathrm{Mpc}^{-1}$, where the detailed shape of halo profiles plays no role (Valageas 2013) and our modelling of the matter density power spectrum is reliable. In agreement with Fig. 1, the deviations from the $\Lambda \mathrm{CDM}$ power spectrum increase with $\left|f_{R_{0}}\right|$ and in the order B4 $>\mathrm{A} 3>\mathrm{E} 3>\mathrm{C} 1$. For the $f(R)$ theories the deviations are smaller for a greater exponent $n$, because this gives a faster increase of the scalar field mass $m$ at higher redshift, as seen in equation (15), whence a faster convergence to $\Lambda \mathrm{CDM}$.
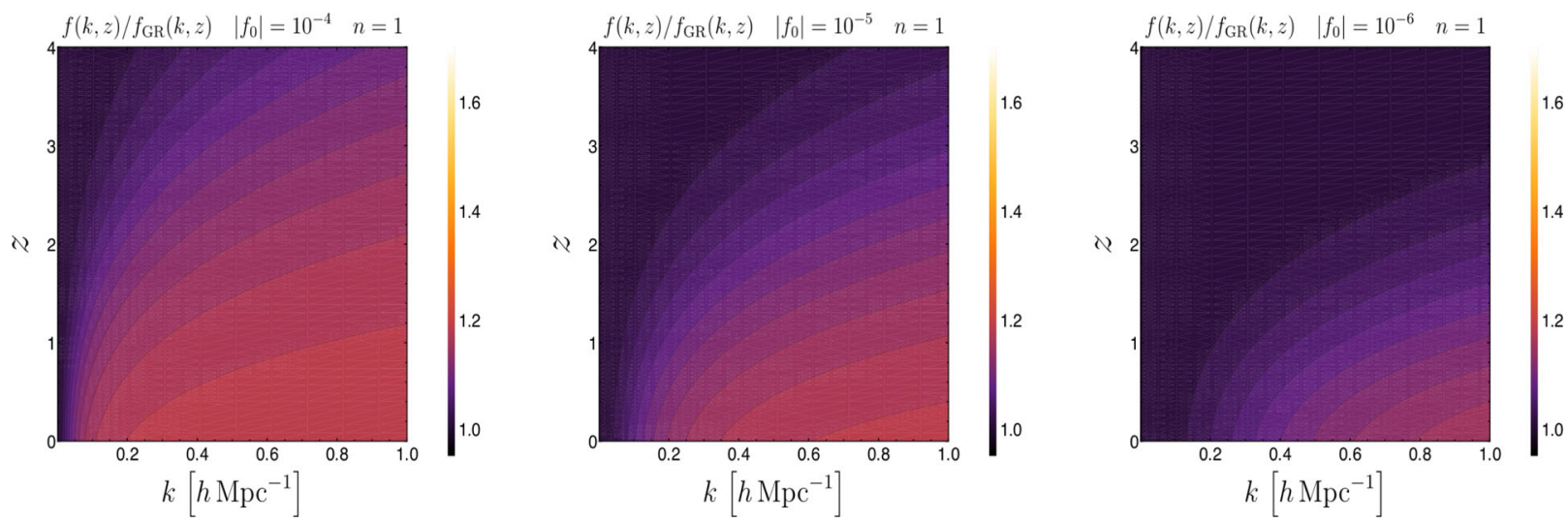

Figure 1. We plot the linear growth rate $f(k, z)=\partial \ln D_{+}(k, z) / \partial \ln a$, normalized to the $\Lambda$ CDM value, for $f(\mathrm{R})$ models as a function of the wavenumber $k$ (in units of $h \mathrm{Mpc}^{-1}$ ) and of the redshift $z$. The functional form for the $f(R)$ model is described in equation (13), with $n=1$. The parameter $f_{R_{0}}$ is fixed at $-10^{-4}$ (left-hand panel), $-10^{-5}$ (middle panel) and $-10^{-6}$ (right-hand panel), respectively. 

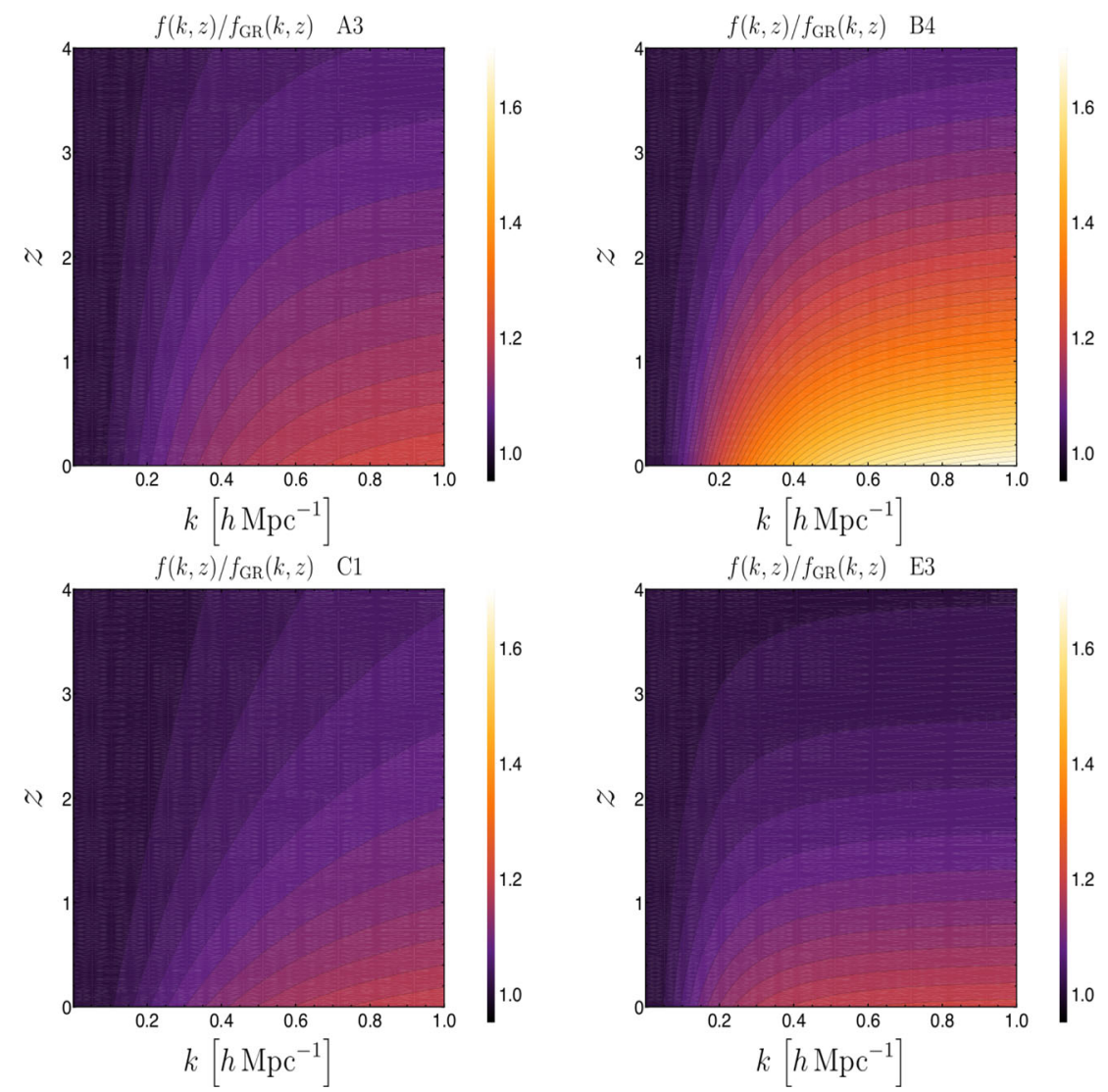

Figure 2. We plot the linear growth rate $f(k, z)=\partial \ln D_{+}(k, z) / \partial \ln a$, normalized to the $\Lambda$ CDM value, as in Fig. 1 but for the Dilaton models. The top panels correspond to the Dilaton models A3 (left) and B4 (right) models. The bottom panels correspond to the Dilaton models C1 (left) and E3 (right), respectively (i.e. only the extreme case from each class of models is shown).
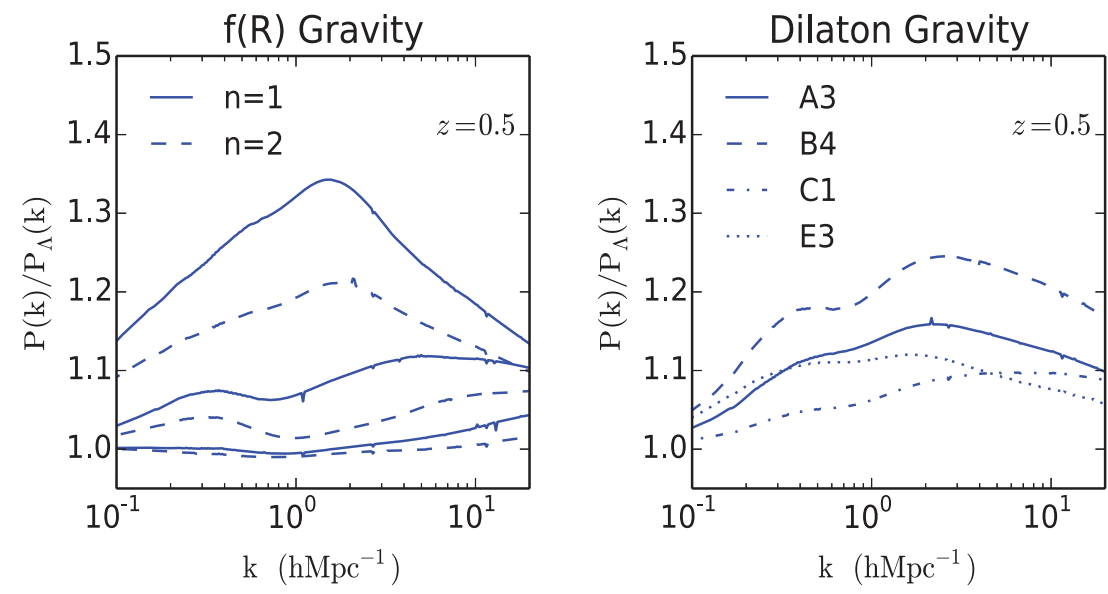

Figure 3. The ratio of the matter power spectra in MG theories and in the $\Lambda \mathrm{CDM}$ model is plotted as a function of the wavenumber $k$. The left-hand panel shows the results for $f(R)$ gravity and the right-hand panel for Dilaton gravity models. The redshift in both panels is fixed at $z=0.5$. We show two different models of $f(R)$ gravity that correspond to $n=1$ (solid lines) and $n=2$ (dashed lines). For each model we consider the three different amplitudes $f_{R_{0}}=-10^{-4},-10^{-5}$ and $-10^{-6}$ (from top to bottom), respectively. The parameters of the Dilaton models displayed in the right-hand panel are given in Table 1. Four different models are shown. Only the extreme version (i.e. with the greatest deviation from $\Lambda \mathrm{CDM}$ ) of each type of model is shown. 


\section{LINEAR RSD (BEYOND KAISER EFFECT) IN MG}

\section{1 sFB formalism}

\subsubsection{Definition of the $S F B$ transform}

Spherical coordinates are often a natural choice in the analysis of cosmological data sets as they can, by an appropriate choice of coordinates, be used to place the observer at the origin of the analysis. As upcoming surveys promise to yield both large (i.e. wide angle) and deep (i.e. large radial coverage) coverage of the sky, we require a simultaneous treatment of the extended radial coverage and spherical sky geometry. A natural basis for such an analysis is given by the sFB formalism. In this section, we follow (Heavens 2003; Castro et al. 2005; Rassat \& Refregier 2012; Pratten \& Munshi 2013) and detail the conventions used in this paper.

Consider a homogeneous 3D random field $\Psi(r, \hat{\Omega})$ such that $\hat{\Omega}$ denotes the angular coordinate on the surface of a sphere and $r$ denotes the comoving radial distance. The eigenfunctions of the Laplacian will be constructed from products of the spherical Bessel functions of the first kind $j_{\ell}(k r)$ and spherical harmonics $Y_{\ell m}(\hat{\Omega})$ with eigenvalues of $-k^{2}$, for a 2 -sphere. Assuming a flat background Universe, the sFB decomposition of the homogeneous 3D field will be given by

$\Psi(r, \hat{\Omega})=\sqrt{\frac{2}{\pi}} \int_{0}^{\infty} \mathrm{d} k \sum_{\{\ell m\}} \Psi_{\ell m}(k) k j_{\ell}(k r) Y_{\ell m}(\hat{\Omega})$,

with the inverse relation given by

$\Psi_{\ell m}(k)=\sqrt{\frac{2}{\pi}} \int_{0}^{\infty} \mathrm{d} r r^{2} \int \mathrm{d} \hat{\Omega} \Psi(r, \hat{\Omega}) k j_{\ell}(k r) Y_{\ell m}^{*}(\hat{\Omega})$.

This is something of a spherical polar analogue to the conventional Cartesian Fourier decomposition. In particular, defining the normalization of the 3D Fourier transform and power spectrum as

$\Psi(\boldsymbol{r})=\frac{1}{(2 \pi)^{3 / 2}} \int \mathrm{d} \boldsymbol{k} \mathrm{e}^{\mathrm{i} i \boldsymbol{k} \cdot \boldsymbol{r}} \Psi(\boldsymbol{k}), \quad\left\langle\Psi(\boldsymbol{k}) \Psi^{*}\left(\boldsymbol{k}^{\prime}\right)\right\rangle=P(k) \delta_{D}\left(\boldsymbol{k}-\boldsymbol{k}^{\prime}\right)$,

the sFB coefficients and the Fourier modes can be related as

$\Psi_{\ell m}(k)=i^{\ell} k \int \mathrm{d} \hat{\Omega} Y_{\ell m}^{*}(\hat{\Omega}) \Psi(k, \hat{\Omega}), \quad \Psi(k, \hat{\Omega})=\frac{1}{k} \sum_{\{\ell m\}}(-i)^{\ell} \Psi_{\ell m}(k) Y_{\ell m}(\hat{\Omega})$,

while the two power spectra obey

$\left\langle\Psi_{\ell m}(k) \Psi_{\ell^{\prime} m^{\prime}}^{*}\left(k^{\prime}\right)\right\rangle=\mathcal{C}_{\ell}(k) \delta_{D}\left(k-k^{\prime}\right) \delta_{\ell \ell^{\prime}} \delta_{m m^{\prime}} \quad$ with $\quad \mathcal{C}_{\ell}(k)=P(k)$.

\subsubsection{Finite-depth surveys}

In reality, we will often want to consider a cosmological random field that is only partially observed due to a finite survey volume. In this instance, we can construct the observed field $\tilde{\Psi}(r, \hat{\Omega})$, which we denote with a tilde, by multiplying the original field $\Psi(r, \hat{\Omega})$ with a selection function $\varphi(r)$

$\tilde{\Psi}(r, \hat{\Omega})=\Psi(r, \hat{\Omega}) \varphi(r)$.

The sFB coefficients of this finite-depth field can be related to those of the field $\Psi$ by

$\tilde{\Psi}_{\ell m}(k)=\int_{0}^{\infty} \mathrm{d} k^{\prime} W_{\ell}\left(k, k^{\prime}\right) \Psi_{\ell m}\left(k^{\prime}\right) \quad$ with $\quad W_{\ell}\left(k, k^{\prime}\right)=\frac{2}{\pi} \int_{0}^{\infty} \mathrm{d} r r^{2} \varphi(r) k j_{\ell}(k r) k^{\prime} j_{\ell}\left(k^{\prime} r\right)$.

The introduction of the selection function means that the homogeneity criterion is not valid in the radial direction and the observed sFB power spectrum will now be given by

$\left\langle\tilde{\Psi}_{\ell m}(k) \tilde{\Psi}_{\ell^{\prime} m^{\prime}}^{*}\left(k^{\prime}\right)\right\rangle=\tilde{\mathcal{C}_{\ell}}\left(k, k^{\prime}\right) \delta_{\ell \ell^{\prime}} \delta_{m m^{\prime}}$, with $\tilde{\mathcal{C}_{\ell}}\left(k, k^{\prime}\right)=\int_{0}^{\infty} \mathrm{d} k^{\prime \prime} W_{\ell}\left(k, k^{\prime \prime}\right) W_{\ell}\left(k^{\prime}, k^{\prime \prime}\right) P\left(k^{\prime \prime}\right)$.

Typically we will often be interested in the diagonal modes for which $k=k^{\prime}$ as the sFB power spectrum falls off rapidly away from the diagonal.

\section{2 sFB formalism: applications}

As previously mentioned, the 3D approach will be particularly important for the analysis of cosmological data from future wide-field surveys. This is especially true for large angular scales in which the plane-parallel approximation, the distant observer approximation or, equivalently, the high $\ell$ approximations to the spherical harmonics are all inadequate. Conventionally, RSD are typically studied in 3D (Fisher et al. 1995) whereas weak lensing has traditionally been studied with projected surveys (2D) due to a lack of, or uncertain, photometric redshift 
information about individual sources. Due to the information available, many of these studies were also limited to small patches of the sky and therefore invoked the flat-sky approximation. With the advent of surveys that can provide accurate photometric redshift information across wide areas of the sky, there has been a growing demand for techniques that can make use of this full-sky 3D information. For example, an early approach to incorporating this 3D information into the 2D projected surveys was to invoke some form of tomographic reconstruction in which the sources are divided up into slices at different redshifts and a 2D analysis is performed in each of these redshift bins. More recently, Heavens (2003) proposed a genuine 3D formalism for weak-lensing surveys based on the sFB expansions. These studies were later extended to a detailed description of weak-lensing observables on the full 3D sky (Castro et al. 2005). In addition, power-spectrum estimation techniques have been generalized to the analysis of higher order statistics in 3D (Munshi, Heavens \& Coles 2011a; Munshi et al. 2011b).

Another growing area of research within the 3D approach is the cross-correlation of galaxy and weak-lensing surveys with other cosmological observables. For example, Shapiro et al. (2012) studied the cross-correlation of 3D galaxy surveys with the projected CMB in order to study the ISW effect. Likewise, Pratten \& Munshi (2014) detailed the cross-correlation of 3D weak lensing with the projected thermal Sunyaev-Zel'dovich effect as a way to recover redshift information that is lost in the line-of-sight projection of the thermal pressure of free elections.

The 3D approach can also be used to study and characterize BAO features in the matter power spectrum. The sFB approach was first used in this context by Rassat \& Refregier (2012) and later extended to include linear RSD as well as non-linear effects in the matter power spectrum by Pratten \& Munshi (2013).

\subsection{Redshift-space distortions}

\subsubsection{Expansion to first order over peculiar velocities}

The existence of inhomogeneous structure in the Universe induces peculiar velocities that lead to distortions in the observed clustering of galaxies as measured in redshift space. The anisotropies generated by these distortions are known as redshift-space distortions (RSD) which, together with bias and non-linear evolution, induce departures in the measured matter power spectrum away from the configuration-space power spectrum predicted by linear perturbation theory (Kaiser 1987). These distortions necessarily complicate the cosmological interpretation of spectroscopic galaxy surveys but the RSD are also one of the most promising probes for the measurement of the growth rate of structure formation and hence a useful probe for models of dark energy and modified theories of gravity.

The effect of the RSD on the matter power spectrum and clustering statistics can be broadly split into two effects: the linear Kaiser effect and the finger of God (FoG) effect. The linear Kaiser effect is a coherent distortion of the peculiar velocity along our line of sight with an amplitude controlled by the growth rate. The Kaiser effect leads to an enhancement of the power-spectrum amplitude at small $k$ (Kaiser 1987). The FoG effect arises due to the random distribution of peculiar velocities for galaxies within virialized structures. These peculiar velocities lead to an incoherent contribution in which we have dephasing and a suppression of the clustering amplitude at high $k$ (Jackson 1972). The effect of a peculiar velocity, or departure from the Hubble flow, $\boldsymbol{v}(\boldsymbol{r})$ at $\boldsymbol{r}$ is to distort the observed comoving position in redshift space $s$ from its true comoving position in real space $r$ :

$\boldsymbol{s}(\boldsymbol{r})=\boldsymbol{r}+\frac{\boldsymbol{v}(\boldsymbol{r}) \cdot \hat{\Omega}}{a H}$

In the following, we denote by a superscript 's' fields that are defined in redshift space $s$, to distinguish them from the real-space fields. The redshift-space $\mathrm{sFB}$ transform can still be defined as in the real-space case,

$\Psi_{\ell m}^{\mathrm{s}}(k)=\sqrt{\frac{2}{\pi}} \int_{0}^{\infty} \mathrm{d} s s^{2} \int \mathrm{d} \hat{\Omega} \Psi^{\mathrm{s}}(s, \hat{\Omega}) k j_{\ell}(k s) Y_{\ell m}^{*}(\hat{\Omega})$.

The conservation of matter implies $\left[1+\delta^{\mathrm{s}}(\boldsymbol{s})\right] \mathrm{d} \boldsymbol{s}=[1+\delta(\boldsymbol{r})] \mathrm{d} \boldsymbol{r}$, so that in the case of the sFB transform of the density contrast we can make the change of integration variable from $\boldsymbol{s}$ to $\boldsymbol{r}$, as

$\delta_{\ell m}^{\mathrm{s}}(k)=\sqrt{\frac{2}{\pi}} \int_{0}^{\infty} \mathrm{d} r r^{2} \int \mathrm{d} \hat{\Omega}[1+\delta(r, \hat{\Omega})] k j_{\ell}(k s) Y_{\ell m}^{*}(\hat{\Omega})-\sqrt{\frac{2}{\pi}} \int_{0}^{\infty} \mathrm{d} s s^{2} \int \mathrm{d} \hat{\Omega} k j_{\ell}(k s) Y_{\ell m}^{*}(\hat{\Omega})$.

The last term only contributes for the monopole $(\ell=m=0)$. Expanding over the peculiar velocity, we obtain

$\{\ell, m\} \neq\{0,0\}: \quad \delta_{\ell m}^{\mathrm{s}}(k)=\sqrt{\frac{2}{\pi}} \int_{0}^{\infty} \mathrm{d} r r^{2} \int \mathrm{d} \hat{\Omega}[1+\delta(r, \hat{\Omega})]\left[k j_{\ell}(k r)+\frac{\boldsymbol{v}(\boldsymbol{r}) \cdot \hat{\Omega}}{a H} k^{2} j_{\ell}^{\prime}(k r)+\cdots\right] Y_{\ell m}^{*}(\hat{\Omega})$.

Using the following perturbative expansion over powers of the peculiar velocity:

$\delta_{\ell m}^{\mathrm{s}}(k)=\delta_{\ell m}^{\mathrm{s}(0)}(k)+\delta_{\ell m}^{\mathrm{s}(1)}(k)+\cdots$,

we have

$\{\ell, m\} \neq\{0,0\}: \quad \delta_{\ell m}^{\mathrm{s}(0)}(k)=\delta_{\ell m}(k)=\sqrt{\frac{2}{\pi}} \int_{0}^{\infty} \mathrm{d} r r^{2} \int \mathrm{d} \hat{\Omega} \delta(r, \hat{\Omega}) k j_{\ell}(k r) Y_{\ell m}^{*}(\hat{\Omega})=i^{\ell} k \int \mathrm{d} \hat{\Omega} \delta(k, \hat{\Omega}) Y_{\ell m}^{*}(\hat{\Omega})$ 
and

$\delta_{\ell m}^{\mathrm{s}(1)}(k)=\sqrt{\frac{2}{\pi}} \int_{0}^{\infty} \mathrm{d} r r^{2} \int \mathrm{d} \hat{\Omega}[1+\delta(r, \hat{\Omega})] \frac{\boldsymbol{v}(\boldsymbol{r}) \cdot \hat{\Omega}}{a H} k^{2} j_{\ell}^{\prime}(k r) Y_{\ell m}^{*}(\hat{\Omega})$.

The continuity equation reads as $a \partial \delta / \partial t+\nabla[(1+\delta) v]=0$. Even though $(1+\delta) v$ is not curl-free, to obtain an order of magnitude estimate we may write

$(1+\delta) v \simeq-a \nabla^{-1} \frac{\partial \delta}{\partial t}$,

which is exact at linear order, and

$$
\begin{aligned}
\delta_{\ell m}^{\mathrm{s}(1)}(k) & \simeq-\sqrt{\frac{2}{\pi}} \int_{0}^{\infty} \mathrm{d} r r^{2} \int \mathrm{d} \hat{\Omega}\left(\nabla^{-1} \frac{\partial \delta}{\partial t}\right)(r, \hat{\Omega}) \cdot \frac{\hat{\Omega}}{H} k^{2} j_{\ell}^{\prime}(k r) Y_{\ell m}^{*}(\hat{\Omega}) \\
& \simeq \frac{2}{\pi} i^{\ell} \frac{k^{2}}{H} \int_{0}^{\infty} \mathrm{d} k^{\prime} k^{\prime} \int \mathrm{d} \hat{\Omega} \frac{\partial \delta\left(k^{\prime}, \hat{\Omega}\right)}{\partial t} \int_{0}^{\infty} \mathrm{d} r r^{2} j_{\ell}^{\prime}(k r) j_{\ell}^{\prime}\left(k^{\prime} r\right) Y_{\ell m}^{*}(\hat{\Omega}) .
\end{aligned}
$$

In the linear regime, the growth rate of the linear growing mode $D_{+}(k, t)$ (which usually depends on the wavenumber in $\mathrm{MG}$ scenarios) is defined as

$f(k, t)=\frac{\partial \ln D_{+}}{\partial \ln a}=\frac{1}{2} \frac{\partial \ln P_{\mathrm{L}}}{\partial \ln a}, \quad$ and $\quad \frac{\partial \delta_{\mathrm{L}}(\boldsymbol{k}, t)}{\partial t}=f H \delta_{\mathrm{L}}$.

In a similar fashion, we define the non-linear growing mode $D_{\mathrm{P}}(k, t)$ and growth rate $f_{\mathrm{P}}(k, t)$ from the non-linear power spectrum as

$D_{\mathrm{P}}(k, t)=\sqrt{\frac{P(k, z)}{P_{\mathrm{L}}(k, 0)}}, \quad f_{\mathrm{P}}(k, t)=\frac{1}{2} \frac{\partial \ln P}{\partial \ln a}$,

where $P_{\mathrm{L}}$ and $P$ are the linear and non-linear power spectra, and we use in equation (35) the approximation

$\frac{\partial \delta(\boldsymbol{k}, t)}{\partial t} \simeq f_{\mathrm{P}} H \delta$,

which is exact at linear order. This gives

$\delta_{\ell m}^{\mathrm{s}(1)}(k) \simeq \frac{2}{\pi} i^{\ell} k^{2} \int_{0}^{\infty} \mathrm{d} k^{\prime} k^{\prime} \int \mathrm{d} \hat{\Omega} Y_{\ell m}^{*}(\hat{\Omega}) \int_{0}^{\infty} \mathrm{d} r r^{2} \delta\left(k^{\prime}, \hat{\Omega}\right) f_{\mathrm{P}}\left(k^{\prime}, z\right) j_{\ell}^{\prime}(k r) j_{\ell}^{\prime}\left(k^{\prime} r\right)$.

Thus, equations (31) and (39) give the exact expression of the matter density sFB transform in redshift space at linear order. Moreover, it includes a partial account of non-linear contributions. The zeroth-order term equation (31) over velocities includes all non-linear contributions from gravitational clustering. The first-order term equation (39) over velocities is exact at linear order but only approximate at non-linear order, because of the approximations equations (33) and (38). Nevertheless, they should capture the magnitude of non-linear contributions to this large-scale Kaiser effect.

Although we go beyond the usual linear-order approximation, by taking into account these non-linear contributions, we restrict ourselves to large weakly non-linear scales, because we neglected small-scale virial motions associated with the FoG effect. Thus, the RSD considered in this paper correspond to the Kaiser effect associated with large-scale coherent flows.

\subsubsection{Finite-depth surveys}

In the case of finite-depth surveys, as in Section 3.1.2, we need to multiply the density field by the selection function $\varphi(r)$. We still define the selection function in real space rather than redshift space because it is not necessarily affected by peculiar velocities in the same fashion as the radial coordinate. Typically, the selection function depends on the flux and observed angular size of the objects and writing it as a function of redshift is a convenient approximation. Besides, it typically varies on cosmological scales, of order $c / H$, which are much greater than the weakly non-linear scales that we aim to probe (or order $2 \pi / k$ ). Then, one can write $\varphi(r) \simeq \varphi(s)$, which also corresponds to neglecting logarithmic radial gradients of the selection function as compared with logarithmic gradients of the density field.

Then, equation (29) becomes

$\{\ell, m\} \neq\{0,0\}: \quad \tilde{\delta}_{\ell m}^{\mathrm{s}}(k)=\sqrt{\frac{2}{\pi}} \int_{0}^{\infty} \mathrm{d} r r^{2} \int \mathrm{d} \hat{\Omega}[1+\delta(r, \hat{\Omega})] \varphi(r)\left[k j_{\ell}(k r)+\frac{\boldsymbol{v}(\boldsymbol{r}) \cdot \hat{\Omega}}{a H} k^{2} j_{\ell}^{\prime}(k r)+\cdots\right] Y_{\ell m}^{*}(\hat{\Omega})$.

We can again expand over the peculiar velocity as in equation (30). The zeroth-order component is given by the real-space expression (24),

$\tilde{\delta}_{\ell m}^{\mathrm{s}(0)}(k)=\int_{0}^{\infty} \mathrm{d} k^{\prime} W_{\ell}^{\mathrm{s}(0)}\left(k, k^{\prime}\right) \delta_{\mathrm{L} \ell m}\left(k^{\prime}, 0\right) \quad$ with

$W_{\ell}^{\mathrm{s}(0)}\left(k, k^{\prime}\right)=\frac{2}{\pi} \int_{0}^{\infty} \mathrm{d} r r^{2} D_{\mathrm{P}}\left(k^{\prime}, z\right) \varphi(r) k j_{\ell}(k r) k^{\prime} j_{\ell}\left(k^{\prime} r\right)$ 
where we defined $\delta_{\mathrm{L} \ell m}\left(k^{\prime}, 0\right)$ the linear density contrast today, at $z=0$, and $D_{\mathrm{P}}(k, z)$ is the non-linear growing mode defined in equation (37), while the first-order component reads as

$\tilde{\delta}_{\ell m}^{\mathrm{s}(1)}(k)=\int_{0}^{\infty} \mathrm{d} k^{\prime} W_{\ell}^{\mathrm{s}(1)}\left(k, k^{\prime}\right) \delta_{\mathrm{L} \ell m}\left(k^{\prime}, 0\right) \quad$ with $\quad W_{\ell}^{\mathrm{s}(1)}\left(k, k^{\prime}\right)=\frac{2}{\pi} k^{2} \int_{0}^{\infty} \mathrm{d} r r^{2} D_{\mathrm{P}}\left(k^{\prime}, z\right) f_{\mathrm{P}}\left(k^{\prime}, z\right) \varphi(r) j_{\ell}^{\prime}(k r) j_{\ell}^{\prime}\left(k^{\prime} r\right)$.

We again define the auto- and cross-power spectra constructed from these harmonic coefficients as

$\left\langle\tilde{\delta}_{\ell m}^{\mathrm{s}(\alpha)}(k) \tilde{\delta}_{\ell^{\prime} m^{\prime}}^{\mathrm{s}(\beta) *}\left(k^{\prime}\right)\right\rangle=\tilde{\mathcal{C}}_{\ell}^{\mathrm{s}(\alpha, \beta)}\left(k, k^{\prime}\right) \delta_{\ell \ell^{\prime}} \delta_{m m^{\prime}}$,

where the indices $\alpha$ and $\beta$ take values $\{0,1\}$, associated with the first two orders of the expansion over peculiar velocities. From equations (42) and (43) we obtain

$\tilde{\mathcal{C}}_{\ell}^{\mathrm{s}(\alpha, \beta)}\left(k, k^{\prime}\right)=\int \mathrm{d} k^{\prime \prime} W_{\ell}^{\mathrm{s}(\alpha)}\left(k, k^{\prime \prime}\right) W_{\ell}^{\mathrm{s}(\beta)}\left(k^{\prime}, k^{\prime \prime}\right) P_{\mathrm{L}}\left(k^{\prime \prime}, 0\right)$,

and, up to first order over peculiar velocities, the redshifted power spectrum reads as

$\tilde{\mathbf{C}}_{\ell}^{\mathrm{s}}\left(k, k^{\prime}\right)=\tilde{\mathcal{C}}_{\ell}^{\mathrm{s}(00)}\left(k, k^{\prime}\right)+\tilde{\mathcal{C}}_{\ell}^{\mathrm{s}(10)}\left(k, k^{\prime}\right)+\tilde{\mathcal{C}}_{\ell}^{\mathrm{s}(01)}\left(k, k^{\prime}\right)$.

The covariance matrix $\tilde{\mathbf{C}}_{\ell}^{\mathrm{s}}\left(k, k^{\prime}\right)$ is symmetric and approximately block diagonal in nature.

The kernels $W_{\ell}^{\mathrm{s}(\alpha)}$ are exact at linear order over the matter density fluctuations and contain some non-linear contributions. Thus, the real-space power $\tilde{\mathcal{C}}_{\ell}^{\mathrm{s}(0,0)}$ is exact at full non-linear order, within the model that we use to compute the non-linear matter power spectrum, but the cross-power $\tilde{\mathcal{C}}_{\ell}^{\mathrm{s}(0,0)}$ is only exact up to linear order, because it involves the approximations equations (33) and (38). To check that the non-linear contributions do not play a significant role, and do not degrade our predictions, we compare our results with those associated with the linear power spectrum itself. Thus, we introduce the kernels $W_{\mathrm{L} \ell}^{\mathrm{s}(0)}$ and $W_{\mathrm{L} \ell}^{\mathrm{s}(1)}$ that source the $3 \mathrm{D}$ linear power spectrum $P_{\mathrm{L}}\left(k^{\prime}, 0\right)$ and depend on the linear growth rate $D_{+}$, respectively, through the following convolutions:

$W_{\mathrm{L} \ell}^{\mathrm{s}(0)}\left(k, k^{\prime}\right)=\frac{2}{\pi} \int_{0}^{\infty} \mathrm{d} r r^{2} D_{+}\left(k^{\prime}, z\right) \varphi(r) k j_{\ell}(k r) k^{\prime} j_{\ell}\left(k^{\prime} r\right)$

$W_{\mathrm{L} \ell}^{\mathrm{s}(1)}\left(k, k^{\prime}\right)=\frac{2}{\pi} k^{2} \int \mathrm{d} r r^{2} D_{+}\left(k^{\prime}, z\right) f\left(k^{\prime}, z\right) \varphi(r) j_{\ell}^{\prime}(k r) j_{\ell}^{\prime}\left(k^{\prime} r\right)$.

These are the linear counterparts of the kernels $W_{\ell}^{\mathrm{s}(0)}$ and $W_{\ell}^{\mathrm{s}(1)}$ defined in equations (42) and (43). The corresponding sFB linear power spectrum is defined through the following equation:

$\tilde{\mathcal{C}}_{\mathrm{L} \ell}^{\mathrm{s}(\alpha, \beta)}\left(k, k^{\prime}\right)=\int \mathrm{d} k^{\prime \prime} W_{\mathrm{L} \ell}^{\mathrm{s}(\alpha)}\left(k, k^{\prime \prime}\right) W_{\mathrm{L} \ell}^{\mathrm{s}(\beta)}\left(k^{\prime}, k^{\prime \prime}\right) P_{\mathrm{L}}\left(k^{\prime \prime}, 0\right)$.

\subsubsection{Galaxy bias}

In practice, we do not observe the matter density field itself, except in weak-lensing surveys, but the galaxy distribution. Writing the galaxy density field as a linear function of the matter density field with a scale-independent bias, $\delta_{\mathrm{g}}(\boldsymbol{r}, z)=b(z) \delta(\boldsymbol{r}, z)$, while the velocity field remains unbiased, we recover the same expressions as in the previous section, but with the kernels $W_{\ell}^{\mathrm{gs}(\alpha)}$ given by

$W_{\ell}^{\mathrm{gs}(0)}\left(k, k^{\prime}\right)=b(z) W_{\ell}^{\mathrm{s}(0)}\left(k, k^{\prime}\right), \quad W_{\ell}^{\mathrm{gs}(1)}\left(k, k^{\prime}\right)=W_{\ell}^{\mathrm{s}(1)}\left(k, k^{\prime}\right)$,

where again the first-order term $W_{\ell}^{\mathrm{gs}(1)}$ is only exact up to linear order over the density and velocity fluctuations. The same relations hold between the linear kernels $W_{\mathrm{L} \ell}^{\mathrm{gs}(\alpha)}$ and $W_{\mathrm{L} \ell}^{\mathrm{s}(\alpha)}$. Then, the expansion (46) is also a first-order expansion over the ratio $\beta_{\mathrm{P}}=f_{\mathrm{P}} / b$.

\section{RESULTS}

If we ignore the effects introduced by the selection function, i.e. set $\varphi(r)=1$, and neglect RSD, i.e. set $s=r$, then we recover the result of Castro et al. (2005) for the un-redshifted contributions $\mathcal{C}_{\ell}(k, k)=P_{\delta \delta}(k)$. These expressions hold for surveys with all-sky coverage. In the presence of homogeneity and isotropy, the 3D power spectrum will be independent of the angular multipole $\ell$. The introduction of a sky mask breaks isotropy and introduces additional mode-mode couplings. The machinery for dealing with partial sky coverage and a sky mask is reviewed in the appendix of Pratten \& Munshi (2013). Note that in the above equations we neglect a number of additional non-linear terms. These include General Relativistic corrections, velocity terms as well as lensing contributions. In addition, the flat sky limits of equation (45) can be found in Pratten \& Munshi (2013).

For $\Lambda \mathrm{CDM}, \beta(k, z) \approx \beta(z)$ but in modified theories of gravity $\beta(k, z)=f(k, z) / b(z)$ is typically $k$-dependant. For both $\Lambda \mathrm{CDM}$ and the modified theories of gravity considered in this paper, we use the full $\beta_{\mathrm{P}}(k, z)$ taken from the numerical calculations of the non-linear matter power spectrum.

We choose a survey with selection function $\varphi(r) \propto \exp \left(-r^{2} / r_{0}^{2}\right)$ and $r_{0}=150 h^{-1} \mathrm{Mpc}$. For the bias we use the model $b(z)=\sqrt{1+z}$. However, we find that our results are not very sensitive to $b(z)$ for the survey configuration that we have considered. The results are presented for all-sky coverage. 

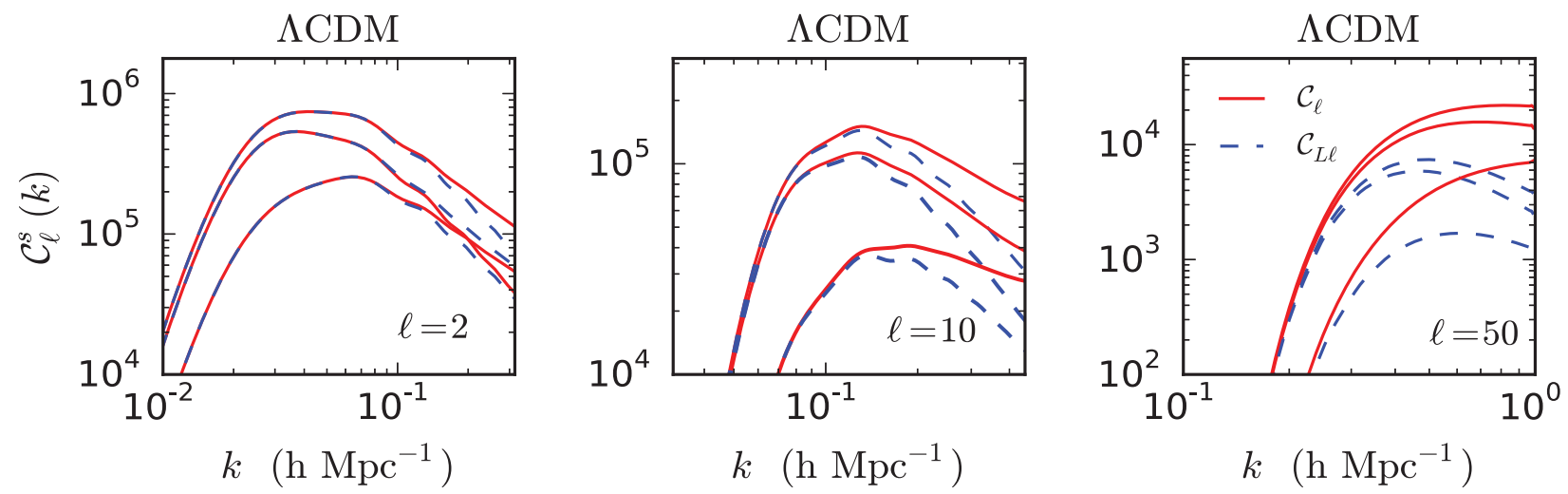

Figure 4. The $3 \mathrm{D}$ power spectra $\tilde{\mathcal{C}}_{\ell}^{\mathrm{s}}=\tilde{\mathcal{C}}_{\ell}^{\mathrm{s}(00)}+\tilde{\mathcal{C}}_{\ell}^{\mathrm{s}(10)}+\tilde{\mathcal{C}}_{\ell}^{\mathrm{s}(01)}$ [in units of $\left.\left(h^{-1} \mathrm{Mpc}\right)^{4}\right]$ for $\Lambda \mathrm{CDM}$ are shown as a function of the radial wavenumber $k$ for various angular harmonics. From left to right the panels represent $\ell=2,10$ and 50, respectively. The topmost curve in each panel corresponds to $\tilde{\mathcal{C}}_{\ell}^{\mathrm{s}}(k, k)$, from equation (46). Next, the two sets of curves, from top to bottom, correspond to the contributions from $\tilde{\mathcal{C}}_{\ell}^{\mathrm{s}(00)}$ and $\tilde{\mathcal{C}}_{\ell}^{\mathrm{s}(10)}+\tilde{\mathcal{C}}_{\ell}^{\mathrm{s}(01)}$, respectively. In addition to the non-linear power $\tilde{\mathcal{C}}_{\ell}^{\mathrm{s}}(k, k)$ (solid lines) defined by the window function (45), we also show the linear power $\tilde{\mathcal{C}}_{\mathrm{L} \ell}^{\mathrm{s}}(k, k)$ (lower dashed lines) defined by the window function (49).

\subsection{Impact of non-linear contributions and of RSD}

We show the diagonal entries of the 3D sFB power spectrum $\tilde{\mathcal{C}}_{\ell}^{\mathrm{s}}(k, k)$, as defined in equation (46), in Fig. 4, for the $\Lambda \mathrm{CDM}$ cosmology. We also plot separately the contributions $\tilde{\mathcal{C}}_{\ell}^{\mathrm{s}(00)}$ (equal to the real-space power) and $\tilde{\mathcal{C}}_{\ell}^{\mathrm{s}(10)}+\tilde{\mathcal{C}}_{\ell}^{\mathrm{s}(01)}$ (the redshift-space contribution at first order over the peculiar velocity power spectrum). In each case we also display the linear power spectra defined by equation (49).

We find that the non-linear contributions are quite small below $k \lesssim 0.2 h \mathrm{Mpc}^{-1}$ but cannot be neglected at higher $k$. This transition scale also corresponds to the harmonic $\ell \gtrsim 10$. For higher $\ell$ the non-linear contribution becomes more pronounced at lower $k$. The RSD typically give a 20 per cent contribution to the total power on the scales displayed in Fig. 4.

\subsection{Off-diagonal terms of the power-spectrum matrix $\tilde{\mathrm{C}}_{\ell}^{\mathrm{s}}\left(k, \boldsymbol{k}^{\prime}\right)$}

A few slices through the window functions $W_{\ell}^{\mathrm{gs}(0)}\left(k, k^{\prime}\right)$ and $W_{\ell}^{\mathrm{gs}(1)}\left(k, k^{\prime}\right)$ that represent mode-mixing in the presence of the radial selection function are displayed in Fig. 5 for $\ell=2$ and Fig. 6 for $\ell=10$. For higher $\ell$ and $k^{\prime}$ the mode mixing is more suppressed, that is, the window function is more strongly peaked around $k^{\prime} \simeq k$.

The power spectra are diagonally dominated. However, a few off-diagonal terms of the matrix $\tilde{\mathbf{C}}_{\ell}^{\mathrm{s}}\left(k, k^{\prime}\right)$ are displayed in Fig. 7 (for $\ell=$ 2) and Fig. 8 (for $\ell=10$ ). The left-hand and right-hand panels show $\tilde{\mathcal{C}}_{\ell}^{\mathrm{s}(00)}$ and $\tilde{\mathcal{C}}_{\ell}^{\mathrm{s}(10)}+\tilde{\mathcal{C}}_{\ell}^{\mathrm{s}(01)}$, respectively. For higher $k^{\prime}$ the covariance matrix is more sharply peaked at $k=k^{\prime}$. The slices through the covariance matrix for $\ell=10$ as depicted in Fig. 8 are more sharply peaked compared to the $\ell=2$ results for the same values of $k^{\prime}$. These properties follow from the behaviour of the window functions $W_{\ell}^{\mathrm{s}}\left(k, k^{\prime}\right)$ displayed in Figs 5 and 6. To compare the diagonal elements with the off-diagonal terms we have also shown $\tilde{\mathcal{C}_{\ell}^{\mathrm{s}}}(k, k)$ in these plots (dashed lines).

\subsection{Impact of the MG models}

In Fig. 9 we plot the diagonal entries of the $3 \mathrm{D} \mathrm{sFB}$ power spectrum $\tilde{\mathcal{C}}_{\ell}^{\mathrm{s}}(k, k)$, as defined in equation (46), as a function of the wavenumber $k$ for $n=1, f(R)$ gravity theories. The results for $n=2$ exhibit similar characteristics. The results correspond to $\ell=2,10$ and 50 , respectively.

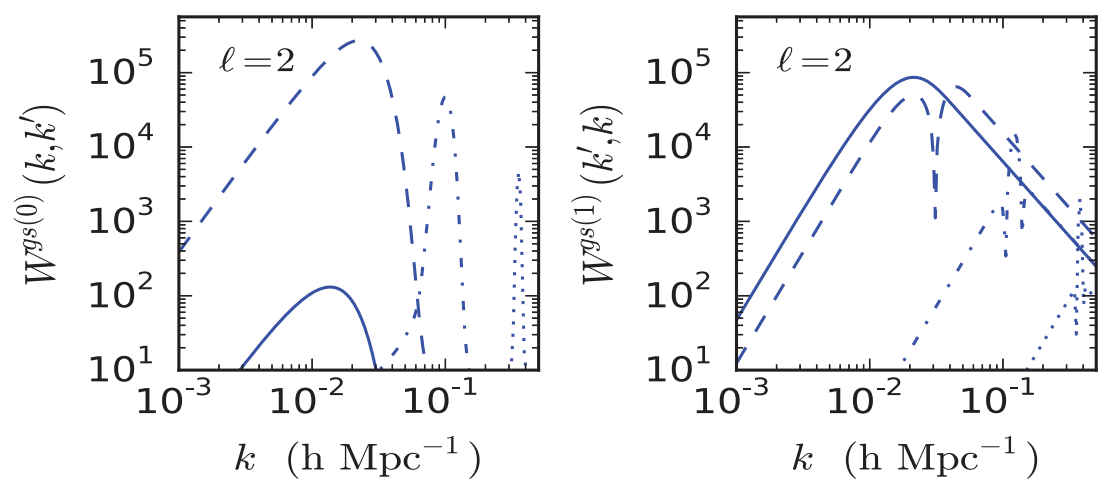

Figure 5. The kernel functions introduced in equation (50) are presented [in units of $\left(h^{-1} \mathrm{Mpc}\right)$ ]. The left-hand panel shows $W^{\mathrm{gs}(0)}\left(k, k^{\prime}\right)$ and the right-hand panel $W^{\mathrm{gs}(1)}\left(k, k^{\prime}\right)$ for $\ell=2$. Different curves with peak position shifting from left to right correspond to $k^{\prime}=$ 0.001 (solid lines), 0.025 (dashed lines), 0.1 (dot-dashed lines), 0.35 (dotted lines) $h \mathrm{Mpc}^{-1}$. 

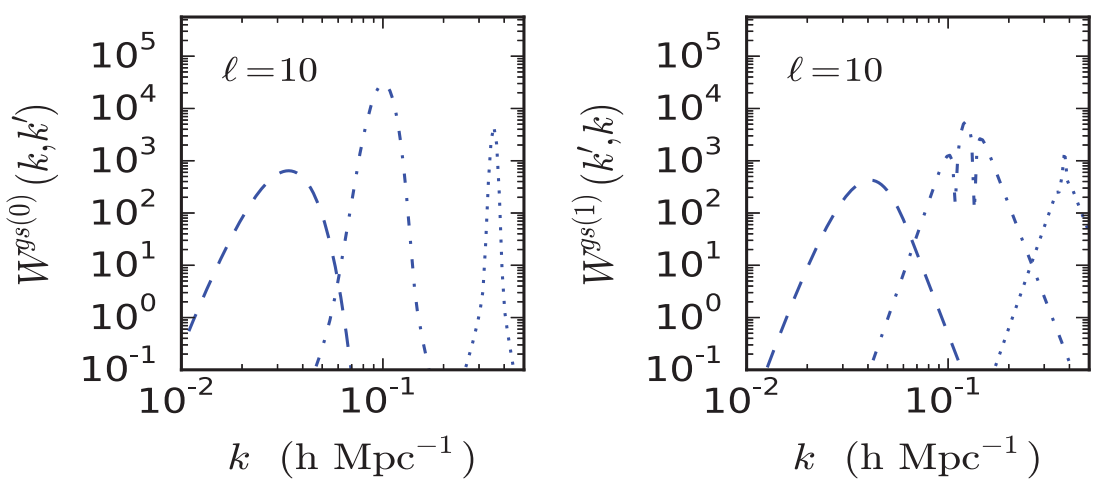

Figure 6. Same as Fig. 5 but for $\ell=10$.
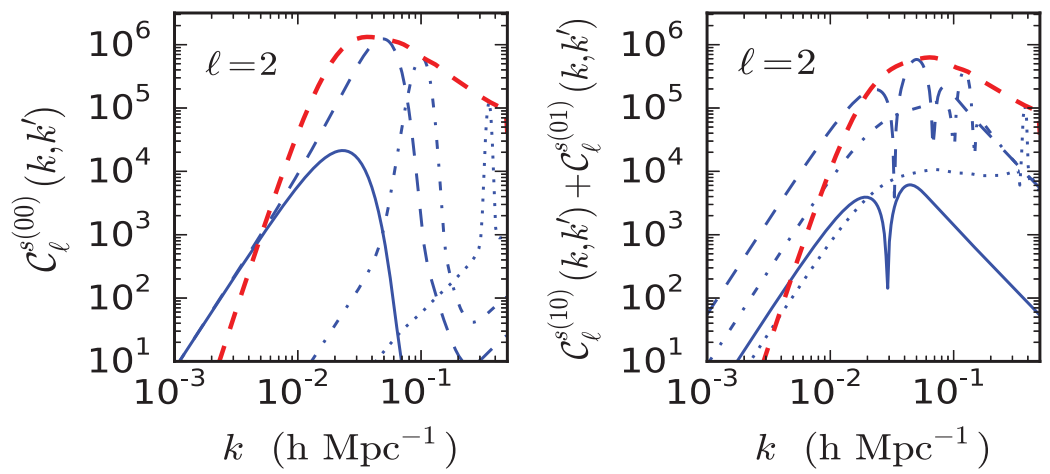

Figure 7. The slices of the covariance matrices are shown for $\ell=2$. The left-hand panel shows $\tilde{\mathcal{C}}_{\ell}^{\mathrm{s}(00)}\left(k, k^{\prime}\right)\left[\right.$ in units of $\left.\left(\mathrm{h}^{-1} \mathrm{Mpc}\right)^{4}\right]$ as a function of $k$ for fixed values of $k^{\prime}$. The right-hand panel corresponds to $\tilde{\mathcal{C}}_{\ell}^{\mathrm{s}(10)}\left(k, k^{\prime}\right)+\tilde{\mathcal{C}}_{\ell}^{\mathrm{s}(01)}\left(k, k^{\prime}\right)$ [in units of $\left(\mathrm{h}^{-1} \mathrm{Mpc}\right)^{4}$ ]. Various solid curves represent different fixed values of $k^{\prime}$, with $k^{\prime}=0.005$ (solid lines), 0.05 (dashed lines), 0.1 (dot-dashed lines) and $0.35 \mathrm{~h} \mathrm{Mpc}^{-1}$ (dotted lines) from left to right. The thick dashed lines in each panel represent the diagonal entries of the covariance matrices, $\tilde{\mathcal{C}}_{\ell}^{\mathrm{s}}(k, k)$.
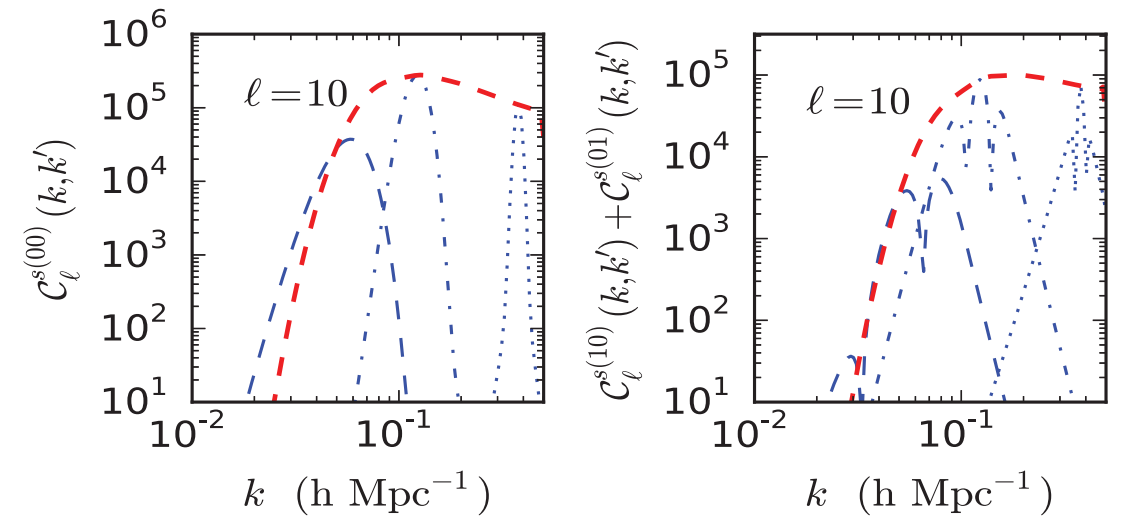

Figure 8. Same as Fig. 7 but for $\ell=10$ and with $k^{\prime}=0.05$ (dashed lines), 0.1 (dot-dashed lines) and 0.35 (dotted lines) $h \mathrm{Mpc}^{-1}$ from left to right.

The range of $k$-values probed is $0.01-0.2 h \mathrm{Mpc}^{-1}$. For each value of $n$ we show the three values $\left|f_{R_{0}}\right|=\left\{10^{-4}, 10^{-5}, 10^{-6}\right\}$. The base $\Lambda$ CDM model is also plotted (solid lines). In comparison to the $\Lambda \mathrm{CDM}$ model all $f(R)$ models have additional power at all $k$. We find that for all values of $k$ and $\ell$ the redshift-space contribution $\mathcal{C}_{\ell}^{\mathrm{s}(10)}+\mathcal{C}_{\ell}^{\mathrm{s}(01)}$ is positive. We will see that the inclusion of redshift information improves our ability to distinguish departures of MG theories from GR. The Dilaton models we consider are specified by the four parameters $m_{0}, r, \beta_{0}$ and $s$, as given in Table 1. The results for $\ell=2$ and 50 are presented in Figs 10 and 11, respectively. In agreement with the 3D power spectra shown in Fig. 3, over the range $k \leq 1 \mathrm{~h} \mathrm{Mpc}^{-1}$ and $\ell \leq 50$ associated with linear and weakly non-linear scales, the relative deviations from the $\Lambda \mathrm{CDM}$ power grow at higher radial wavenumber $k$ and angular harmonic $\ell$. 

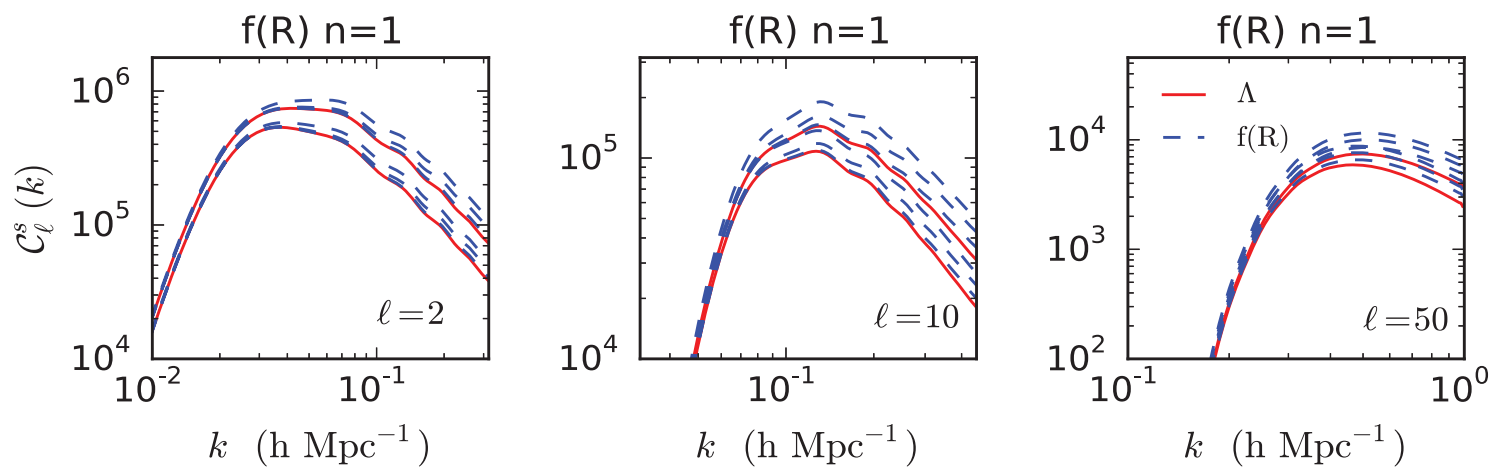

Figure 9. The $3 \mathrm{D}$ linear power spectra $\tilde{\mathcal{C}}_{\mathrm{L} \ell}^{\mathrm{s}}(k)\left[\right.$ in units of $\left.\left(h^{-1} \mathrm{Mpc}\right)^{4}\right]$ that represent the diagonal elements of the $3 \mathrm{D}$ sFB power spectrum $\tilde{\mathcal{C}}_{\mathrm{L} \ell}^{\mathrm{s}}(k, k)$ as defined in equation (46) for $f(R), n=1$, theories of gravity are plotted as a function of the radial wavenumber $k$ for $\ell=2$ (left-hand panel), $\ell=10$ (middle panel) and $\ell=50$ (right-hand panel), respectively. In each panel the dashed curves correspond to the three different parameter values $f_{R_{0}}=-10^{-4},-10^{-5}$ and $-10^{-6}$ (from top to bottom). The $\Lambda \mathrm{CDM}$ result is shown with solid curves. Each panel displays two different sets of curves. The top and lower sets correspond to sFB power spectra $\tilde{\mathcal{C}}_{\mathrm{L} \ell}^{\mathrm{s}}(k)$ with and without the RSD.
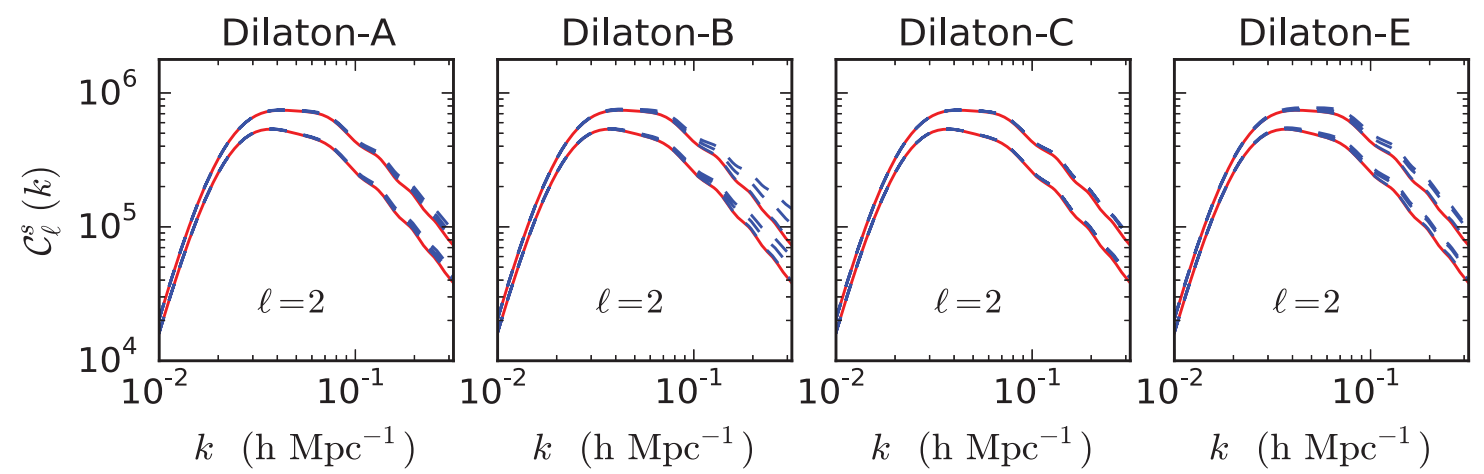

Figure 10. The 3D linear power spectra $\tilde{\mathcal{C}}_{\mathrm{L} \ell}^{\mathrm{s}}(k)$, as in Fig. 9 but for the Dilaton models [in units of $\left.\left(h^{-1} \mathrm{Mpc}\right)^{4}\right]$, are shown as a function of the radial wavenumber $k$ for the angular harmonic $\ell=2$. In each panel the solid curves represent the $\Lambda$ CDM result while the dashed curves represent the Dilaton models. From left to right the panels correspond to the Dilaton models A, B, C and E, respectively. The upper (lower) set of curves in each panel corresponds to results with (without) RSD. For each chosen Dilaton gravity model we plot the power spectra for the three choices of parameter values given in Table 1 . The models $\mathrm{A}, \mathrm{B}, \mathrm{C}$ and $\mathrm{E}$ correspond to variation of the parameters $s, \beta_{0}, r$ and $m_{0}$, respectively.

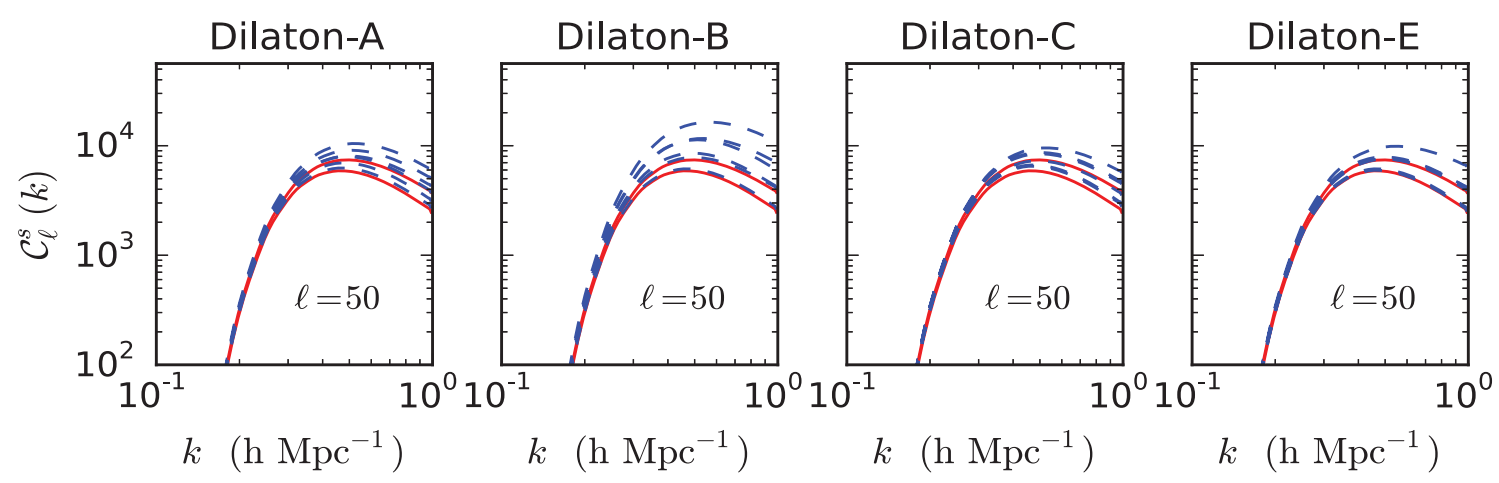

Figure 11. Same as Fig. 10 but for $\ell=50$.

\section{COVARIANCE AND $\chi^{2}$}

The likelihood function $\mathcal{L}$ for arbitrary sets of parameter $\Theta_{\mu}$ [that specify a given MG theory; e.g. $\left\{n, f_{R_{0}}\right\}$ in the case of $f(R)$ theory], given the data vector $\tilde{\boldsymbol{C}}_{\ell}^{\mathrm{s}}(k)$ [which consists of the noisy $3 \mathrm{D}$ sFB power-spectra $\tilde{\boldsymbol{C}}_{\ell}^{\mathrm{s}}(k) \equiv \tilde{\mathcal{C}}_{\ell}^{\mathrm{s}}(k, k)$ ], is given by

$\mathcal{L}\left(\Theta_{\mu} \mid \tilde{\boldsymbol{C}}_{\ell}^{\mathrm{s}}(k)\right)=\frac{1}{(2 \pi)^{N_{\mathrm{pix}} / 2}|\operatorname{det} \mathbf{C}|^{1 / 2}} \exp \left[-\frac{1}{2} \sum_{\ell \ell^{\prime}} \int \mathrm{d} k \int \mathrm{d} k^{\prime} \delta \mathcal{C}_{\ell}^{\mathrm{s}}(k) \mathbf{C}_{\ell \ell^{\prime}}^{-1}\left(k, k^{\prime}\right) \delta \mathcal{C}_{\ell^{\prime}}^{\mathrm{s}}\left(k^{\prime}\right)\right]$. 

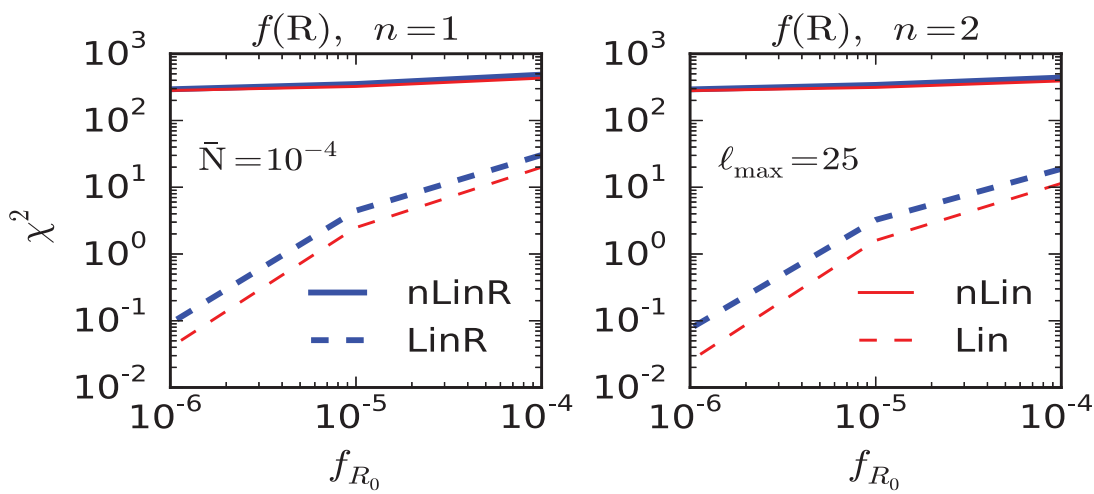

Figure 12. The $\chi^{2}$ for the $f(R)$ models are presented as a function of the parameter $\left|f_{R_{0}}\right|$. The left-hand panel corresponds to $n=1$ and the right-hand panel to $n=2$. The two solid curves near the top of the panels correspond to results using non-linear power spectrum (equation 45 ). The curve with higher $\chi^{2}$ for a given $f_{R_{0}}$ includes RSD contribution (nLinR) while the one (nLin) with lower $\chi^{2}$ does not. The two dashed curves at the bottom of the panel are the results derived using linear power spectrum (equation 49) with and without RSD contribution denoted as LinR and Lin, respectively. Inclusion of RSD improves the $\chi^{2}$ in both linear and non-linear regime.

Here $\delta \tilde{\mathcal{C}}_{\ell}^{\mathrm{s}}=\tilde{\mathcal{C}_{\ell}^{\mathrm{s}}}-\tilde{\mathcal{C}}_{\ell}^{\mathrm{sGR}}\left(\mathcal{C}_{\ell}^{\mathrm{sGR}}\right.$ being the power spectrum in GR); $N_{\text {pix }}$ is the size of the data vector, which depends on the angular resolution $\ell_{\max }$ and the number of radial bins used in the computation. The covariance matrix $\mathbf{C}_{\ell \ell^{\prime}}\left(k, k^{\prime}\right)$ is given by

$\mathbf{C}_{\ell \ell^{\prime}}\left(k, k^{\prime}\right) \equiv\left\langle\tilde{\mathcal{C}}_{\ell}^{\mathrm{s}}(k) \tilde{\mathcal{C}}_{\ell^{\prime}}^{s}\left(k^{\prime}\right)\right\rangle-\left\langle\tilde{\mathcal{C}}_{\ell}^{\mathrm{s}}(k)\right\rangle\left\langle\tilde{\mathcal{C}}_{\ell^{\prime}}^{\mathrm{s}}\left(k^{\prime}\right)\right\rangle=\frac{2}{2 \ell+1}\left[\tilde{\mathcal{C}}_{\ell}^{\mathrm{s}}\left(k, k^{\prime}\right)+\frac{1}{\bar{N}}\right]^{2} \delta_{\ell \ell^{\prime}} \delta_{m m^{\prime}}$,

where we used a Gaussian approximation. Here $\bar{N}$ represents the number density of galaxies which are assumed to be Poisson distributed. The block diagonal form is a result of assuming an all-sky coverage. However we found the $\mathbf{C}$ to be diagonally dominant. Partial sky coverage will introduce off-diagonal terms between different harmonics in the covariance matrix. A rough scaling of signal-to-noise (S/N) with the fraction of sky-coverage $f_{\text {sky }}$ is typically used in the literature: $\mathrm{S} / \mathrm{N} \propto \sqrt{f_{\text {sky }}}$. We work with the $\chi^{2}$ statistics defined as

$\chi^{2}=\sum_{\ell \ell^{\prime}} \int \mathrm{d} k \int \mathrm{d} k^{\prime} \delta \tilde{\boldsymbol{C}}_{\ell}^{\mathrm{s}}(k) \mathbf{C}_{\ell \ell^{\prime}}^{-1}\left(k, k^{\prime}\right) \delta \tilde{\mathcal{C}}_{\ell^{\prime}}^{\mathrm{s}}\left(k^{\prime}\right)$

We assume a perfect knowledge of all background cosmological parameters and present results with and without RSD. In practice, we bin the radial wavenumber $k$ in four logarithmic bins to avoid the covariance matrix being singular, thus replacing the integrals over $k$ and $k^{\prime}$ by discrete sums, and we use the multipoles $\ell=1-25$. The galaxy number density is fixed at $\bar{N}=10^{-4} \mathrm{Mpc}^{-3}$. We again assume all-sky coverage and the survey depth is fixed at $r_{0}=150 h^{-1} \mathrm{Mpc}$. We show our results for the $f(R)$ theories in Fig. 12. For the $n=1$ model we find that for $\bar{N}=10^{-4} \mathrm{Mpc}^{-3}$ the values of $\left|f_{R_{0}}\right|>2 \times 10^{-5}$ can be ruled out with a $3 \sigma$ confidence. For $n=2$ we find the constraint degrades to $\left|f_{R_{0}}\right|>3 \times 10^{-5}$. The $f(R)$ models with higher exponent $n$ are progressively less constrained as they converge increasingly fast to $\Lambda$ CDM with redshift at $z>0$. We show our results for the Dilaton models in Fig. 13. We consider the problem of the estimation of each individual parameter $\left\{s, \beta_{0}, r, m_{0}\right\}$ while keeping others fixed, which corresponds to the model families A, B, C and E. For $\bar{N}=10^{-4} \mathrm{Mpc}^{-3}$ we find that the parameter values $\beta_{0}>0.8$ and $m_{0}<0.15 \mathrm{~h} \mathrm{Mpc}^{-1}$ can be ruled out with $3 \sigma$ confidence. No meaningful constraints on $\sigma$ or $r$ can be obtained at $3 \sigma$ level. Clearly, a joint estimation will be more demanding. These parameters will also have some degeneracy with the parameters describing the background cosmological dynamics. A joint Fisher analysis of MG parameters and cosmological parameters will require a dedicated study and will be presented elsewhere. As the Planck observations provide an accurate baseline standard cosmological model, using Planck prior may be a useful practical solution.
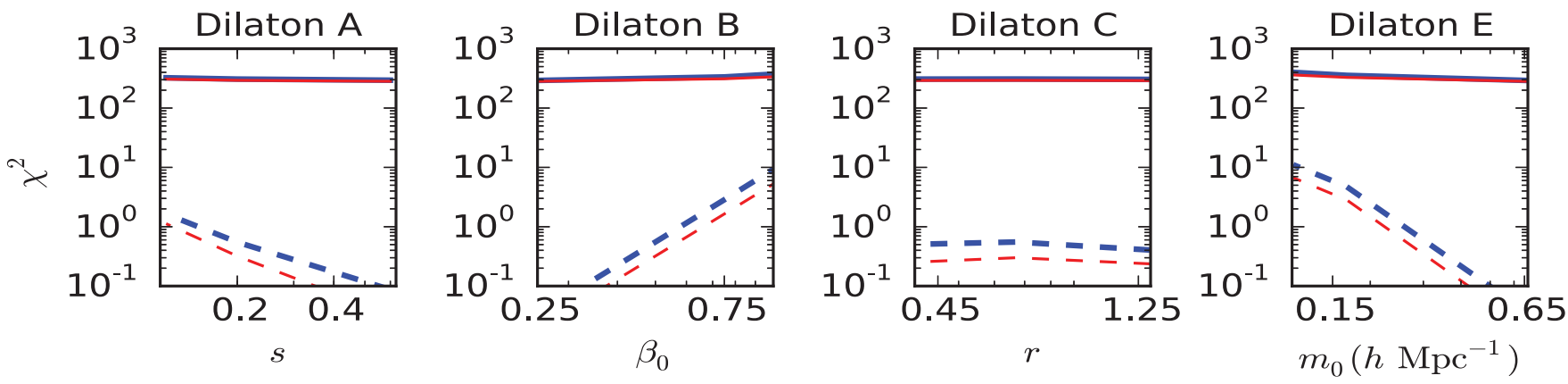

Figure 13. The $\chi^{2}$ for various generalized Dilaton models (see Table 1) are shown. From left to right various panels correspond to models A, B, C and E. Each model corresponds to variation of a specific parameter among $\left\{s, \beta_{0}, r, m_{0}\right\}$ while keeping all other parameters fixed. The $\chi^{2}$ in panels from left to right is shown as a function of $s, \beta_{0}, r$ and $m_{0}$. The line-style is the same as in Fig. 12. 
In the near future, the Dark Energy Spectroscopic Instrument (DESI; formerly BIGBOSS) plans to cover $14000 \mathrm{deg}^{2}\left(f_{\text {sky }}=1 / 3\right)$ yielding 30 million targets. The Subaru telescope Prime Focus Spectrograph ${ }^{11}$ (PFS), is due to have first light in 2017. The current cosmology design envisages a survey cover of $1500 \mathrm{deg}^{2}$ yielding three million galaxies. For DESI we expect $f_{R_{0}}>9 \times 10^{-5}\left(10^{-4}\right)$ for $n=1(2)$ models to be ruled out with $2 \sigma$ confidence level. However, due to the low fraction of sky coverage, similar constraints from PFS are expected to be less competitive against existing cosmological probes. Notice that, observations by SDSS galaxy surveys in the low to moderate redshift range $0.15<z<0.67$ is already being used to set interesting constraints on any departure from GR, e.g. Bel et al. $(2015)$ obtained $f_{R_{0}}<4.6 \times 10^{-5}$ at the $2 \sigma$ level. We would also like to stress that our results are based on all-sky analysis and we use an $f_{\text {sky }}$ scaling to take into account the effect of partial sky coverage. Surveys that cover a small fraction of sky such as PFS will have to deal with strong mode-mode coupling that drives the covariance matrix to near singularity. A detailed analysis will be presented elsewhere. Our constraints are derived for a spectroscopic survey. Inclusion of photometric redshift error will degrade the discriminating power of the survey.

The comparison of the linear and non-linear curves in Figs 12 and 13 suggests that the non-linear contributions can make a significant effect. However, in the non-linear regime the covariance matrix should include the bispectrum and the trispectrum of the density field, which would reduce the $\chi^{2}$. Therefore, we can expect that a fully non-linear analysis, with a better modelling of the covariance matrix (e.g. from numerical simulations) would lower down the non-linear curves and make them closer to the linear result. However, because the departures from the $\Lambda \mathrm{CDM}$ power spectrum increase on weakly non-linear scales, non-linearities should still improve the discriminatory power of the analysis of 3D clustering as compared with the linear result. Here we follow a conservative approach as we estimate the constraints on the MG parameters by using the linear-theory $\chi^{2}$.

We have studied the constraints in both $f(R)$ and Dilaton models with and without RSD. We find that RSD only lead to a small broadening of the constraints. Below galaxy number density of $\bar{N}=10^{-4} \mathrm{Mpc}^{-3}$ the surveys rapidly lose their ability to discriminate. On the other hand, increasing the number density beyond $\bar{N}=10^{-3} \mathrm{Mpc}^{-3}$ does not lead to drastic improvements of the results.

Our constraints are based on lower order tangential modes $\ell \leq 25$. We find that the information contents in different $\ell$ are highly degenerate. In the range $0.01 \leq k \leq 0.2 \mathrm{~h} \mathrm{Mpc}^{-1}$, we find that for more than three to four (logarithmic) bins in $k$ the covariance matrix can become singular.

Finally, our results are based on a uniform distribution of noise (constant $\bar{N}$ ) while real surveys may have more complicated variations in the average galaxy number density which may depend on the radial and angular coordinates in the sky.

\section{DISCUSSION AND FUTURE PROSPECTS}

In this paper we have studied the possibility of constraining MG theories using galaxy clustering. We have studied two different MG models: $f(R)$ theories and Dilaton theories. In both cases we have used a specific parametrization and computed the $\chi^{2}$ as a criterion for constraining the departure from the $\Lambda \mathrm{CDM}$ models. We used the sFB transforms and the resulting 3D power spectrum $\mathcal{C}_{\ell}(k)$ for a range of $\ell$ and $k$ values to constrain the model. We assume an all-sky coverage and a spectroscopic survey with a Gaussian selection function $\varphi(r) \propto \exp \left(-r^{2} / r_{0}^{2}\right), r_{0}=150 h^{-1} \mathrm{Mpc}$. We fix the number density of galaxies to be $\bar{N}=10^{-4} \mathrm{Mpc}^{-3}$. We find that the low $\ell \leq 25 \mathrm{modes}$ of $\mathcal{C}_{\ell}^{\mathrm{s}}\left(k, k^{\prime}\right)$ (with radial modes restricted to $k<0.2 \mathrm{~h} \mathrm{Mpc}^{-1}$ ) can constraint the parameter $f_{R_{0}}$ at a level of $2 \times 10^{-5}\left(3 \times 10^{-5}\right)$ with $3 \sigma$ confidence for $n=1(2)$. For the Dilaton models some of the parameters $\left(\beta_{0}, m_{0}\right)$ can be well constrained using galaxy clustering though there are others $(s, r)$ which remain poorly constrained. The parametrization used by us depends on a tomographic approach. Combining constraints from higher $\ell \geq 25$ modes can further reduce the error-bar and thus in principle make cosmological probes of gravity competitive with Solar system tests. However this will require an accurate modelling of non-linear clustering in redshift space as well as the covariance matrix of the sFB power spectra. Our results are based on the linear power spectrum. However, we find that inclusion of non-linear effect can drastically improve the $\chi^{2}$. However, we would like to emphasize that the contribution from higher order moments notably the trispectrum is not included in our covariance matrix. Although, this may not play an important role in the quasi-linear regime, in the non-linear regime such contributions can no longer be ignored. A detailed analysis will be presented elsewhere. Partial sky coverage, boundary conditions from specific survey geometry will also mean that in a realistic survey the sFB modes will have to be discrete and not continuous even in the radial direction. The average number density of galaxies and hence the noise too will be a function of the angular position on the surface of the sky. A pseudo- $\mathcal{C}_{\ell}$ (Hivon et al. 2002) based approach can be useful in this direction. The errors due to photometric redshift determination can be readily included in our calculation.

We have only considered two sets of MG theories. However, the results derived are quite general and can readily be extended to other MG scenarios such as the K-mouflage models or the Symmetron models. In addition, the sFB power spectrum can also be useful in constraining massive neutrinos, warm dark matter candidates or axionic dark matter through their footprints on the matter power spectrum as a function of redshift.

The number of parameters required to specify an MG model can be high. Non-parametric techniques such as PCA can be efficient to investigate linear combinations (ordered according to decreasing $\mathrm{S} / \mathrm{N}$ ) of parameters that can be extracted using a specific survey strategy. Extending the $\chi^{2}$-based approach presented here, a more extensive analysis of survey optimization covering a range of survey parameter

${ }^{11} \mathrm{http} / / /$ sumire.ipmu.jp/en/2652 
and associated Fisher matrix analysis detailing parameter degeneracies will be presented elsewhere. In future we also plan to use a Bayesian model selection approach based on the evidences for acceptance or rejection of specific models.

Physics of galaxy formation may be different in MG models. Even in the $\Lambda$ CDM cosmology, the bias associated with the galaxies may depend on the galaxy type and their environment, and may be stochastic. Such complications can only be dealt with numerical simulations. However, one may expect that at very large scale such effects may be less important.

CMB mostly probes high redshifts where any modification from GR is expected to be sub-dominant. However, CMB is also sensitive to modifications of gravity through secondary effects such as the ISW effect and the weak-lensing distortions (Planck Collaboration XIV 2015). However the constraints are rather weak compared to what can be achieved by galaxy surveys (Munshi et al. 2014).

Weak lensing is a very exciting possibility for constraining any departure from GR especially because it is free from the problem of galaxy bias (Munshi et al. 2008). However, in recent years many possible systematics have been discussed, e.g. from intrinsic ellipticity correlations or possible modifications of the power spectrum due to Active Galactic Nucleus feedback, gas cooling and stellar feedback or neutrino physics (Munshi et al. 2015, in preparation). Such contaminations may induce appreciable bias in parameter estimation (e.g. Osato, Shirasaki \& Yoshida 2015). Despite all these systematics, weak lensing and galaxy clustering remain the most powerful probe of possible departures from GR on cosmological scales.

Statistics of Lyman $\alpha$ absorption have also been investigated in the context of $f(R)$ gravity (Arnold, Puchwein \& Springel 2015). Using cosmological hydrodynamical simulations of $f(R)$ gravity which include the flux probability distribution functions and the flux power-spectra, and an analysis of the column density and line-width distributions, as well as the matter power spectrum, it was found that Lyman $\alpha$ statistics are rather insensitive to modification to gravity in $f(R)$ models. Hence no competitive constraints are achievable using current data. Moreover, contamination by baryonic physics, associated with star formation and cooling processes, imply that a very accurate modelling of such ingredients is required. Constraints from 21-cm intensity mapping are more encouraging (Hall et al. 2013).

\section{ACKNOWLEDGEMENTS}

DM and PC acknowledge support from the Science and Technology Facilities Council (grant no. ST/L000652/1). The research leading to these results has received funding from the European Research Council under the European Union's Seventh Framework Programme (FP/2007-2013)/ERC Grant Agreement no. [616170]. PV acknowledges support from the French Agence Nationale de la Recherche under Grant ANR-12-BS05-0002. DM would like to thank A. Starobinsky and A. Heavens for useful discussions. It is a pleasure for DM to acknowledge related collaborations with B. Hu, L. van Waerbeke and J. Harnois-Deraps.

\section{REFERENCES}

Abramo L. R., Reimberg P. H., Xavier H. S., 2010, Phys. Rev. D, 82, 043510

Amendola L., Kunz M., Sapone D., 2008, J. Cosmol. Astropart. Phys., 4, 13

Amendola L., Catena R., Masina I., Notari A., Quartin M., Quercellini C., 2011, J. Cosmol. Astropart. Phys., 1107, 027

Arnold C., Puchwein E., Springel V., 2015, MNRAS, 448, 2275

Asorey J., Crocce M., Gaztanaga E., Lewis A., 2012, MNRAS, 427, 1891

Bel J., Brax P., Marinoni C., Valageas P., 2015, Phys. Rev. D, 91, 103503

Bernardeau F., Colombi S., Gaztanaga E., Scoccimarro R., 2002, Phys. Rep., 367, 248

Bertschinger E., 2006, ApJ, 648, 797

Brax P., Valageas P., 2013, Phys. Rev. D, 88, 023527

Brax P., van de Bruck C., Davis A.-C., Shaw D. J., 2008, Phys. Rev. D, 78, 104021

Brax P., Davis A.-C., Li B., Winther H. A., Zhao G.-B., 2012a, J. Cosmol. Astropart. Phys., 10, 002

Brax P., Davis A.-C., Li B., Winther H. A., 2012b, Phys. Rev. D, 86, 044015

Brax P., Davis A.-C., Li B., 2012c, Phys. Lett. B, 715, 38

Buchdahl H. A., 1970, MNRAS, 150, 1

Castro P. G., Heavens A. F., Kitching T. D., 2005, Phys. Rev. D, 72, 023516

Cheung C., Fitzpatrick A. L., Kaplan J., Senatore L., Creminelli P., 2008, J. High Energy Phys., 3,14

Chiba T., 2003, Phys. Lett. B, 575, 1

Clifton T., Ferreira P. G., Padilla A., Skordis C., 2012, Phys. Rep., 513, 1

Damour T., Polyakov A. M., 1994, Nucl. Phys. B, 423, 532

Daniel S. F., Linder E. V., Smith T. L., Caldwell R. R., Cooray A., Leauthaud A., Lombriser L., 2010, Phys. Rev. D, 81, 123508

Dosset J., Hu B., Parkinson D., 2014, J. Cosmol. Astropart. Phys., 1403, 046

Erdogue P. et al., 2006, MNRAS, 368, 1515

Ferreira P. G., Skordisn C., 2010, Phys. Rev. D, 81, 104020

Fisher K. B., Lahav O., Hoffman Y., Lynden-Bell D., Zaroubi S., 1995, MNRAS, 272, 885

Gil-Marin H., Schmidt F., Hu W., Jimenez R., Verde L., 2011, J. Cosmol. Astropart. Phys., 1111, 019

Guzzo L. et al., 2008, Nature, 451, 541

Hall A., Bonvin C., Challinor A., 2013, Phys. Rev. D, 87, 064026

Heavens A., 2003, MNRAS, 343, 1327

Heavens A., Taylor A., 1995, MNRAS, 343, 1327

Heavens A. F., Kitching T. D., Verde L., 2007, MNRAS, 380, 1029

Hinterbichler K., Khoury J., 2010, Phys. Rev. Lett., 104, 231301

Hivon E., Górski K. M., Netterfield C. B., Crill B. P., Prunet S., 2002, ApJ, 567, 2 
Hojjati A., Zhao G. B., Pogosian L., Silvestri A., Crittenden R., Koyama K., 2012, Phys. Rev. D, 85, 043508

Hu W., Sawicki I., 2007b, Phys. Rev. D, 76, 104043

Jackson J. C., 1972, MNRAS, 156, 1P

Jain B., Zhang P., 2008, Phys. Rev. D, 78, 063503

Jain B., Vikram V., Sakstein J., 2013, ApJ, 779, 39

Jennings E., Baugh C. M., Li B., Zhao G. B., Koyama K., 2012, MNRAS, 425, 2128

Joyce A., Jain B., Khoury J., Trodden M., 2015, Phys. Rep., 568, 1

Kaiser N., 1987, MNRAS, 227, 1

Lanusse F., Rassat A., Starck J.-L., 2012, A\&A, 540, 60

Laureijs R. et al., 2011, preprint (arXiv:1110.3193)

Li B., Mota D. F., Barrow J. D., 2011, ApJ, 728, 109

Li B., Hellwing W. A., Koyama K., Zhao G. B., Jennings E., Baugh C. M., 2013, MNRAS, 428, 743

Linder E. V., 2005, Phys. Rev. D., 72, 043529

Lombriser L., Slosar A., Seljak U., Hu W., 2012, Phys. Rev. D, 85, 124038

Munshi D., Valageas P., van Waerbeke L., Heavens A., 2008, Phys. Rep., 462, 67

Munshi D., Heavens A., Coles P., 2011a, MNRAS, 411, 2161

Munshi D., Kitching T., Heavens A., Coles P., 2011b, MNRAS, 416, 629

Munshi B., Hu B., Renzi A., Heavens A., Coles P., 2014, MNRAS, 442, 821

Navarro J. F., Frenk C. S., White S. D. M., 1996, ApJ, 462, 563

Nunez A., Solganik S., 2004, preprint (arXiv:hep-th/0403159)

Olive K. A., Pospelov M., 2008, Phys. Rev. D, 77, 043524

Osato K., Shirasaki M., Yoshida N., 2015, ApJ, 806, 186

Ostriker J. P., Steinhardt P., 2003, Science, 300, 1909

Oyaizu H., Lima M., Hu W., 2008, Phys. Rev. D, 78, 123524

Perlmutter S. et al., 1999, ApJ, 517, 565

Pietroni M., 2005, Phys. Rev. D, 72, 043535

Planck Collaboration XIV, 2015, preprint (arXiv:1502.01590)

Pogosian L., Silvestri A., 2008, Phys. Rev. D, 77, 023503

Pratten G., Munshi D., 2013, MNRAS, 436, 3792

Pratten G., Munshi D., 2014, MNRAS, 442, 759

Rassat A., Refregier A., 2012, A\&A, 540, 115

Rassat A. et al., 2005, preprint (astro-ph/0510195)

Riess A. G. et al., 1998, AJ, 116, 1009

Schimdt F., 2008, Phys. Rev. D, 78, 3002

Schlegel D. J. et al., 2007, Am. Astron. Soc. Meeting Abstr. Vol. 211, p. 132.29

Shapiro C., Crittenden R. G., Percival W. J., 2012, MNRAS, 422, 2341

Song Y.-S., Hu W., Sawicki I., 2007a, Phys. Rev. D, 75, 044004

Song Y.-S., Peiris H., Hu W., 2007b, Phys. Rev. D, 76, 063517

Starobinsky A. A., 1980, Phys. Lett. B, 91, 99

Starobinsky A. A., 2007, JETP Lett., 86, 157

Tsujikawa S., Tatekawa T., 2008, Phys. Lett. B, 665, 325

Tyson J. A., LSST, 2004, Am. Astron. Soc. Meeting Abstr. Vol. 36, p. 108.01

Vainshtein A., 1972, Phys. Lett. B, 39, 393

Valageas P., 2013, Phys. Rev. D, 88, 083524

Valageas P., Nishimichi T., Taruya A., 2013, Phys. Rev. D, 87, 083522

Vikram V., Cabre A., Jain B., VanderPlas J. T., 2013, J. Cosmol. Astropart. Phys., 1308, 020

Zhang P., 2006, Phys. Rev. D, 73, 123504

Zhao G. B., Pogosian L., Silvestri A., Zylberberg J., 2008, Phys. Rev. D, 79, 083513

Zhao G. B., Pogosian L., Silvestri A., Zylberberg J., 2009, Phys. Rev. Lett., 103, 241301

Zhao G. B., Giannantonio T., Pogosian L., Silvestri A., Bacon D. J., Koyama K., Nichol R. C., Song Y. S., 2010, Phys. Rev. D, 81, 103510

Zhao G. B., Li B., Koyama K., 2011, Phys. Rev. D, 83, 044007

This paper has been typeset from a $\mathrm{T}_{\mathrm{E}} \mathrm{X} / \mathrm{L} \mathrm{AT} \mathrm{E} \mathrm{X}$ file prepared by the author. 